\title{
Variety of fiber orientation tensors
}

Mathematics and Mechanics of Solids I-27

(C) The Author(s) 2021

\section{(c) (i) (5)}

Article reuse guidelines:

sagepub.com/journals-permissions DOI: $10.1177 / 10812865211057602$

journals.sagepub.com/home/mms

Julian Karl Bauer

Institute of Mechanics, Karlsruhe Institute of Technology (KIT), Germany

Thomas Böhlke

Institute of Engineering Mechanics, Karlsruhe Institute of Technology (KIT), Germany

Received 5 June 202I; accepted: 16 October 2021

\begin{abstract}
Fiber orientation tensors are established descriptors of fiber orientation states in (thermo-)mechanical material models for fiber-reinforced composites. In this paper, the variety of fourth-order orientation tensors is analyzed and specified by parameterizations and admissible parameter ranges. The combination of parameterizations and admissible parameter ranges allows for studies on the mechanical response of different fiber architectures. Linear invariant decomposition with focus on index symmetry leads to a novel compact hierarchical parameterization, which highlights the central role of the isotropic state. Deviation from the isotropic state is given by a triclinic harmonic tensor with simplified structure in the orientation coordinate system, which is spanned by the second-order orientation tensor. Material symmetries reduce the number of independent parameters. The requirement of positive-semi-definiteness defines admissible ranges of independent parameters. Admissible parameter ranges for transversely isotropic and planar cases are given in a compact closed form and the orthotropic variety is visualized and discussed in detail. Sets of discrete unit vectors, leading to selected orientation states, are given.
\end{abstract}

\section{Keywords}

Fiber orientation tensor, anisotropy, harmonic decomposition, fiber-reinforced composites,

\section{Introduction}

The microstructure of fiber-reinforced composites significantly affects the effective mechanical properties of the compound. Two local microstructure descriptors commonly used to predict the effective properties are the fiber volume fraction and the fiber orientation distribution. The local fiber orientation distribution is completely defined by a fiber orientation distribution function (ODF). However, in practical applications the ODF is usually approximated by a limited number of fiber orientation tensors, as tensor representations fit into the continuum mechanics framework. Multiple kinds of orientation tensors exist [1]. Practical applications related to fiber orientations include both identification of the fiber orientation information by experimental methods [2] or process simulation [3] and usage of the orientation information in fullfield [4], meanfield [5, 6], or damage simulations [7]. Both groups of applications, identification and usage of orientation information, benefit from 
damage simulations [7]. Both groups of applications, identification and usage of orientation information, benefit from a well-known variety of fiber orientation tensors. If the variety of fiber orientation tensors is well defined, the identified orientation tensors can be assessed and the space of input parameters of methods predicting effective mechanical properties is set. The variety of second-order fiber orientation tensors is well known $[8-10]$ and used, e.g., in $[4,11,12]$ to generate clear pictures of the dependence of mechanical properties on the second-order directional measures. However, concise descriptions and parameterizations of fourth-order fiber orientation tensors are rare. The variety of transversely isotropic fourth-order orientation tensors is identified by [13] and used in [5, 14]. [5] showed that the identification of effective mechanical properties based on up to second-order orientation tensors yields insufficient accuracy [15]. For a given second-order orientation tensor, closure approximations $[8,9,16-18]$ identify a corresponding fourth-order orientation tensor based on assumptions. Closure approximations are used by, e.g., [5, 14, 19-21] to model mechanical properties. [15] assesses the reconstruction of ODFs based on closure approximations and minimum entropy method used by [5, 22].

This paper addresses the research question on the variety of fiber orientation tensors of order two and four. The paper has review character and is structured as follows. The definition of ODF and fiber orientation tensors is followed by a review on the variety of second-order orientation tensors, leading to the orientation triangle. The classical parameterization of the orientation triangle is framed and parameterizations which highlight the isotropic state and material symmetry are motivated. Implications of index symmetry of fourth-order tensors in Mandel's [23] notation are discussed, before fourth-order orientation tensors are treated as special Hooke tensors and harmonic decomposition is applied. This translates the reasoning of [1] into continuum mechanics notation, leading to simplified notation with a focus on the isotropic state. A parameterization of a generic fourth-order orientation tensor is given based on a parameterization of the second-order orientation tensor in combination with a triclinic structure tensor. Admissible orientation states are identified by demanding positive semi-definiteness. Results of $[5,13]$ on admissible transversely isotropic orientation tensors are reproduced and framed by coefficient-wise constraints. Minimal sets of discrete fiber orientations leading to special transversely isotropic orientation states are presented and discussed. The variety of orthotropic fourth-order fiber orientation tensors is visualized. Transversely isotropic and planar orthotropic fourth-order orientation tensors are highlighted as limiting orthotropic cases and admissible parameter ranges are specified. A compact parameterization of planar orthotropic fourth-order orientation tensors is given. Minimal sets of discrete planar orthotropic fiber orientations visualize the character of fourth-order orientation information. The variety of planar fourthorder fiber orientation tensors including a parameterization and admissible ranges is given. Notes on closure approximations close this paper. This paper closes with notes on a small set of closure approximations which are contrasted by the variety of fourth-order orientation tensors.

\section{Notation}

Symbolic tensor notation is preferred in this paper. Tensors of first order are denoted by bold lowercase letters such as $\mathbf{q}, \mathbf{n}, \mathbf{v}$, e. Tensors of second order are denoted by bold uppercase letters such as $\mathbf{N}$ or $\mathbf{Q}$ and fourthorder tensors are denoted by bold openface, e.g., $\mathbb{N}$ or $\mathbb{D}$. Tensors in representations for varying tensor order are represented by, e.g., $\mathbb{D}_{\langle k\rangle}$, where $k$ defines the tensor order. A linear mapping of a second-order by a fourth-order tensor reads as $\mathbf{A}=\mathbb{C}[\mathbf{B}]$ and the scalar product reads as $\mathbf{A} \cdot \mathbf{B}$. The tensor power, i.e., the $k$ th dyadic product of, e.g., a first-order tensor $\mathbf{a}$ is denoted by $\mathbf{a}^{\otimes k}$ yielding, e.g., $\mathbf{a}^{\otimes 3}=\mathbf{a} \otimes \mathbf{a} \otimes \mathbf{a}$. An orthonormal basis is denoted by $\left\{\mathbf{e}_{i}\right\}$ with $\mathbf{e}_{i} \cdot \mathbf{e}_{j}=\delta_{i j}$ and the Kronecker delta $\delta_{i j}$. If a matrix of tensor coefficients is used in mixed notation, the coefficient matrix is directly followed by the tensor basis where the first index of the basis corresponds to the rows of the coefficients matrix, the second one to the columns. Summation convention applies, unless otherwise stated. Representations in index notation always refer to an orthonormal basis. The Rayleigh product is used to represent an active rotation of a physical quantity and for a first-order tensor is defined by $\mathbf{Q} \star \mathbf{n}=n_{i} \mathbf{Q} \mathbf{e}_{i}$. Sets, i.e., collections of quantities, are denoted by calligraphic symbols, e.g., $\mathcal{F}$ and are constructed by curly braces. Inside the curly braces, elements of the set are given explicitly, or by a generator expression following the pattern \{quantity $\mid$ condition fulfilled by elements contained in set\}. Although, this work and related code is based on [24, 25], numbering and indices follow the continuum mechanics convention starting at one. 


\section{Variety of fiber orientation tensors}

\section{I. Fiber orientation}

Given a reference volume (RV) of arbitrary size and shape, which might be interpreted as the part of a body $\mathcal{B}$ centered around position $\mathbf{x}$. The orientation of axisymmetric fibers inside this RV can be described by the fiber orientation distribution function (ODF)

$$
\psi: \mathcal{S}^{2} \rightarrow \mathbb{R}, \quad \text { with } \mathcal{S}^{2}=\left\{\mathbf{n} \in \mathbb{R}^{3} \mid\|\mathbf{n}\|=1\right\}
$$

mapping any direction $\mathbf{n}$ onto a scalar value $\psi(\mathbf{n}) . \mathcal{S}^{2}$ is the two-dimensional surface of a unit sphere parameterized by, e.g., a unit vector $\mathbf{n}$. The function $\psi(\mathbf{n})$ is non-negative, i.e.

$$
\psi(\mathbf{n}) \geq 0, \quad \forall \mathbf{n} \in \mathcal{S}^{2}
$$

holds and normalization of $\psi(\mathbf{n})$ implies

$$
\int_{\mathcal{S}^{2}} \psi(\mathbf{n}) \mathrm{d} n=1 .
$$

As fibers have a direction but no attitude, $\psi(\mathbf{n})$ is symmetric, i.e.,

$$
\psi(-\mathbf{n})=\psi(\mathbf{n}), \quad \forall \mathbf{n} \in \mathcal{S}^{2}
$$

holds (see [4, 26]). The fiber orientation distribution function solely describes the orientation of the fibers. Being a one-point statistic information, the ODF contains no additional information on, e.g., the spatial arrangement of the fibers inside the reference volume. In a heterogeneous material with spatially varying microstructure, the ODF usually is influenced by the size of the RV (see, e.g., the size parameter in [27, Figure 4]). If the fibers have identical volumes, e.g., because of identical length and constant cross section, the volume fraction $c_{\mathcal{F}}$ of those fibers pointing through $\mathcal{F} \subseteq \mathcal{S}^{2}$ on all fibers, is given by

$$
c_{\mathcal{F}}=\frac{1}{V_{\mathrm{f}}} \int_{V_{\mathcal{F}}} \mathrm{d} V=\int_{\mathcal{F}} \psi(\mathbf{n}) \mathrm{d} n
$$

with the volume of all fibers $V_{\mathrm{f}}$ and $V_{\mathcal{F}}$ being the volume of fibers pointing through $\mathcal{F}$ (see [5, 28]). Equation (5) demonstrates the transformation of a volume average, e.g., over $V_{\mathcal{F}}$, into an average over corresponding parts of the unit sphere. Volume averages on a reference volume $V_{\mathrm{RV}}$ of a field quantity $\mathbf{q}(\mathbf{x})$ which is axisymmetric about a spatially varying principle axis $\mathbf{n}(\mathbf{x})$ can be transformed into an average over $\mathcal{S}^{2}$

$$
\frac{1}{V_{\mathrm{RV}}} \int_{V_{\mathrm{RV}}} \mathbf{q}(\mathbf{x}) \mathrm{d} V=\int_{\mathcal{S}^{2}} \psi(\mathbf{n}) \mathbf{q}(\mathbf{n}) \mathrm{d} n,
$$

if $\mathbf{q}$ can be parameterized solely in $\mathbf{n}$. Similar reasoning for volume averages over the special orthogonal group $S O(3)$ in context of crystal orientation distribution functions (CODFs) are used extensively in the literature, see, e.g., [22, 29]. ODF averages are limited to axisymmetric quantities because ODF is defined on directions. In contrast, CODF can be interpreted as rotations of coordinate systems and therefore induce no restriction on the quantity which is to be averaged. The ODF reflects the material symmetry of the microstructure. As a consequence,

$$
\psi(\mathbf{S} \star \mathbf{n})=\psi(\mathbf{n}), \quad \forall \mathbf{S} \in S_{\mathrm{RV}} \subseteq S O(3)
$$

holds. Equation (7) implies that $\psi(\mathbf{n})$ is symmetric with respect to all rotations $\mathbf{S}$ which are contained in the symmetry group of the microstructure $S_{\mathrm{RV}}$ being a subset of $S O(3)$. Following [30], eight different material symmetries exist and tensor representations of Hooke tensors following these symmetries are given in, e.g., [31]. An inclusion scheme, depicting the hierarchy of the symmetry classes combined with illustrations of the symmetry planes, can be found in [32, Figure 4]. Implications of material symmetries on second-order tensors are summarized in Appendix A. 


\subsection{Orientation tensors of first kind}

In the standard framework of continuum mechanics, physical quantities are expressed as invariant tensors. This enables the use of known transformation rules and simplifies the storage in computer memory. Kanatani [1] approximates experimentally obtained directional data by tensor series and [26] defines orientation tensors of Kanatani [1] first kind by

$$
\mathbb{N}_{\langle k\rangle}=\int_{\mathcal{S}^{2}} \psi(\mathbf{n}) \mathbf{n}^{\otimes k} \mathrm{~d} n
$$

with $\mathbf{n}^{\otimes k}$ being the $k$ th moment of $\mathbf{n}$. For example, the resulting second- and fourth-order tensors are

$$
\begin{aligned}
& \mathbf{N}=\mathbb{N}_{\langle 2\rangle}=\int_{\mathcal{S}^{2}} \psi(\mathbf{n}) \mathbf{n} \otimes \mathbf{n} \mathrm{d} n \\
& \mathbb{N}=\mathbb{N}_{\langle 4\rangle}=\int_{\mathcal{S}^{2}} \psi(\mathbf{n}) \mathbf{n} \otimes \mathbf{n} \otimes \mathbf{n} \otimes \mathbf{n} \mathrm{d} n .
\end{aligned}
$$

Equation (8) can be interpreted as a weighted summation of moment tensors. The moment tensor represents the tensorial character of a specific direction and weights are specified by the distribution information of the ODF. It follows from Equation (8) that $\mathbf{N}$ and $\mathbb{N}$ are completely symmetric with respect to index permutations. Odd orientation tensors vanish due to the symmetry of $\psi(\mathbf{n})$ and higher-order tensors contain all tensors of lower order as

$$
\mathbb{N}_{\langle k-2\rangle}=\mathbb{N}_{\langle k\rangle}\left[\mathbf{I}_{2}\right]
$$

holds for $2 \leq k$ with the identity on second-order tensors $\mathbf{I}_{2}$. As a consequence of the normalization of both $\psi(\mathbf{n})$ and $\mathbf{n}$, the limiting case of Equation (11) yields

$$
\mathbf{N} \cdot \mathbf{I}_{2}=\operatorname{tr}(\mathbf{N})=1 .
$$

Orientation tensors of Kanatani [1] first kind are commonly used to represent experimentally obtained directional data, e.g., from computer tomography scans or results of flow simulations [27].

\subsection{Orientation tensors of third kind}

The ODF can be expressed as a tensorial Fourier series

$$
\psi(\mathbf{n})=\frac{1}{4 \pi} \sum_{k=0}^{\infty} \hat{\mathbb{D}}_{\langle k\rangle} \cdot \mathbf{n}^{\otimes k}
$$

which is called spherical harmonic expansion [1, page 154] and introduces orientation tensors of Kanatani [1] third kind

$$
\hat{\mathbb{D}}_{\langle k\rangle}=\frac{2 k+1}{2^{k}}\left(\begin{array}{c}
2 k \\
k
\end{array}\right) \operatorname{dev}\left(\mathbb{N}_{\langle k\rangle}\right)
$$

Constructing $\hat{\mathbb{D}}_{\langle k\rangle}$ in Equation (14) contains two steps, first taking the deviatoric part of $\mathbb{N}_{\langle k\rangle}$ and, second, scaling with factors for the series expansion given by $\frac{2 k+1}{2^{k}}\left(\begin{array}{c}2 k \\ k\end{array}\right)$. Skipping the second step, defines orientation tensors of third kind, as done by, e.g., [5], as

$$
\mathbb{D}_{\langle k\rangle}=\operatorname{dev}\left(\mathbb{N}_{\langle k\rangle}\right)
$$


In combination with Equations (11) and (12) this leads for $k \in[2,4]$ to

$$
\begin{aligned}
\mathbb{D}_{\langle 2\rangle}=\operatorname{dev}(\mathbf{N}) & =\mathbf{N}-\frac{1}{3} \mathbf{I}_{2} \operatorname{tr}(\mathbf{N}) \\
& =\mathbf{N}-\mathbf{N}^{\text {iso }} \\
\mathbb{D}_{\langle 4\rangle}=\operatorname{dev}(\mathbb{N}) & =\mathbb{N}-\frac{6}{7} \operatorname{sym}\left(\left(\mathbb{N}\left[\mathbf{I}_{2}\right]\right) \otimes \mathbf{I}_{2}\right)+\frac{3}{35} \operatorname{sym}\left(\mathbf{I}_{2} \otimes \mathbf{I}_{2}\right) \operatorname{tr}\left(\mathbb{N}\left[\mathbf{I}_{2}\right]\right) \\
& =\mathbb{N}-\frac{6}{7} \operatorname{sym}\left(\mathbf{N} \otimes \mathbf{I}_{2}\right)+\frac{3}{35} \operatorname{sym}\left(\mathbf{I}_{2} \otimes \mathbf{I}_{2}\right)
\end{aligned}
$$

with the isotropic orientation tensor of second order

$$
\mathbf{N}^{\text {iso }}=\frac{1}{3} \mathbf{I}_{2} .
$$

The operator $\operatorname{dev}(\cdot)$ extracts the deviatoric part and the operator $\operatorname{sym}(\cdot)$ extracts the totally symmetric part with respect to index symmetry. Definitions of the operators $\operatorname{sym}(\cdot)$ and $\operatorname{dev}(\cdot)$ for higher-order tensors are given in [33]. In other communities, alternative representations of directional data are common. The connection to spherical harmonics in the context of quantitative texture analysis is discussed in $[1,14,15,29]$.

\subsection{Variety of second-order orientation tensors}

Equations (2) and (8) imply that orientation tensors of Kanatani [1] first kind and second order $\mathbf{N}$ are symmetric and positive semi-definite. As a consequence, $\mathbf{N}$ can be diagonalized, i.e., pairs of eigenvalues $\lambda_{i}$ with $\lambda_{i} \geq 0$ and orthonormal eigenvectors $\mathbf{v}_{i}$ for $i \in[1,2,3]$ exist, such that

$$
\mathbf{N}=N_{i j}^{(2)} \mathbf{e}_{i} \otimes \mathbf{e}_{j}=\sum_{i=1}^{3} \lambda_{i} \mathbf{v}_{i} \otimes \mathbf{v}_{i}=\left[\begin{array}{lll}
\lambda_{1} & 0 & 0 \\
& \lambda_{2} & 0 \\
\operatorname{sym} & & \lambda_{3}
\end{array}\right] \mathbf{v}_{i} \otimes \mathbf{v}_{j}
$$

holds and there exists a rotation defined by an orthogonal tensor

$$
\mathbf{Q}=\mathbf{v}_{i} \otimes \mathbf{e}_{i}
$$

mapping the arbitrary but fixed basis $\left\{\mathbf{e}_{i}\right\}$ onto the basis $\left\{\mathbf{v}_{i}\right\}$. In the following, the orthonormal basis $\left\{\mathbf{v}_{i}\right\}$ spanned by the eigenvectors is called the orientation coordinate system. The arbitrary ordering convention of the eigenvalues

$$
\lambda_{3} \leq \lambda_{2} \leq \lambda_{1}
$$

is common in literature (see [21, 23]). The constraint in Equation (12) is equivalent to

$$
\lambda_{1}+\lambda_{2}+\lambda_{3}=1
$$

and reduces the number of independent components of $\mathbf{N}$ from six to five. The coordinate system $\left\{\mathbf{v}_{i}\right\}$ or equivalently the mapping $\mathbf{Q}$ is defined by three scalars, leaving two scalars specifying structural information on fiber orientations inside the orientation coordinate system. As a consequence, the variety of $\mathbf{N}$ can be expressed as a two dimensional space known as the orientation triangle, e.g., parameterized in pairs $\left(\lambda_{1}, \lambda_{2}\right)$, in combination with a mapping $\mathbf{Q}$ which defines the orientation coordinate system. Information of $\mathbf{N}$ is limited to the definition of a coordinate system and two half axes of an ellipsoid aligned with theses coordinate axes, see [10]. The third half axis of the ellipsoid is defined by Equation (24). The weakest material symmetry which can be described by $\mathbf{N}$ is orthotropy (see [10] or Appendix A).

Representations of orientation triangles are given, e.g., in $[8,9,11,21]$ as well as in Figure 1(a). An alternative visualization is given by the orientation invariant map in [9, Figure 1b]. The triangle in Figure 1(a) is only one of the possible orientation triangles and is called the standard orientation triangle. The boundaries of the triangle are labeled in Figure 1(a) and follow directly from Equations (23) and (24) and $0 \leq \lambda_{3}$. Following 


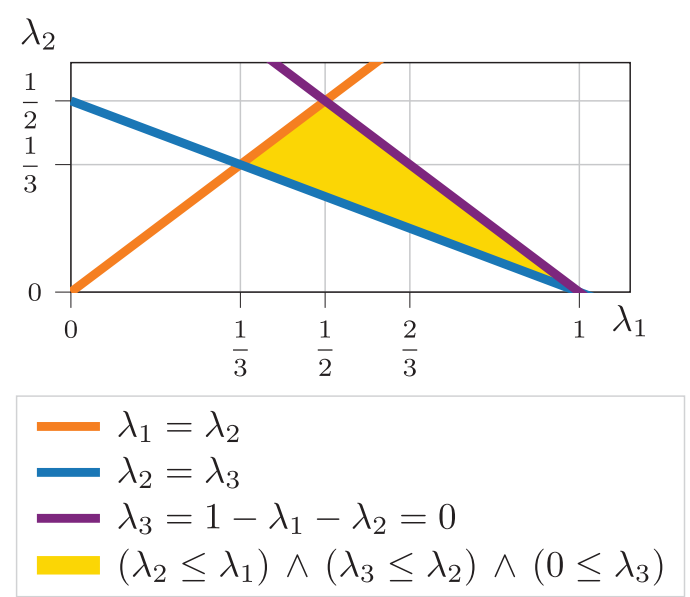

(a)

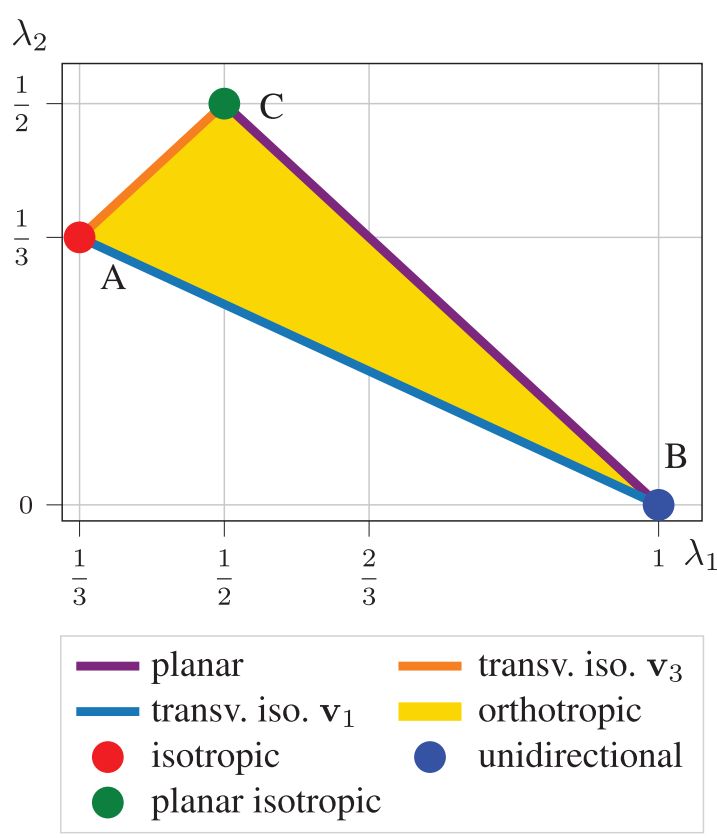

(b)

Figure I. (a) Constraints defining the orientation triangle. (b) Material symmetries in the orientation triangle [8].

[8], vertices and edges of the triangle are discussed, based on Figure 1(b). The vertices $A, B$, and $C$ are given by the extremal orientation states, which are isotropic, unidirectional, and planar isotropic. Starting from a planar isotropic state $\left(\lambda_{1}=\lambda_{2}=1 / 2, \lambda_{3}=0\right)$ at vertex $C$, moving along the orange edge towards vertex $A, \lambda_{3}$ increases whereas $\lambda_{1}$ and $\lambda_{2}$ decrease uniformly until the isotropic state $\left(\lambda_{1}=\lambda_{2}=\lambda_{3}\right)$ is reached. Continuing from vertex $A$ along the edge towards vertex $B$, the largest eigenvalue $\lambda_{1}$ increases while $\lambda_{2}$ and $\lambda_{3}$ decrease uniformly until the unidirectional state $\left(\lambda_{1}=1, \lambda_{2}=\lambda_{3}=0\right)$ is reached. All states along the edges $C A$ and $A B$ have at least two identical eigenvalues and, therefore, are transversely isotropic with principle axes being $\mathbf{v}_{3}$ and $\mathbf{v}_{1}$, respectively. The material symmetries of points of the orientation triangle are visualized in Figure 1(b) and discussions can be found in $[8,10]$. The states along the edge $B C$ are planar as $\lambda_{3}$ vanishes. Starting from vertex $B$ along the edge towards vertex $C, \lambda_{1}$ decreases and $\lambda_{2}$ increases until both are equal. All points inside the triangle including the edge between vertices $B$ and $C$ are orthotropic. In summary, two edges are transversely isotropic, one edge is planar and one point is isotropic.

The triangles in Figures 1(a) and (c) are projections of one sixth of a orientation plane in the threedimensional space spanned by $\left\{\mathbf{v}_{i}\right\}$ given in Figure 2(a). For each point in the orientation triangle, five corresponding points with identical structural properties and different ordering conventions of eigenvalues exist. To illustrate the redundancy which is inherent in the orientation plane, a randomly chosen orientation state with eigenvalues being any permutation of $(1 / 2,1 / 3,1 / 6)$ is marked in orange multiple times in Figure 2(a) and (b). For each eigenvector in $\left\{\mathbf{v}_{i}\right\}$, transversely isotropic orientation states with principle axis $\mathbf{v}_{i}$ are located along a straight line being the intersection of the orientation plane and a plane with $\lambda_{m}=\lambda_{n}$ for $i \neq m \neq n$. Planar states are found at the intersection of the orientation plane and planes of one vanishing eigenvalue. The central character of the isotropic state is reflected by Figure 2(a). The three-dimensional representation in Figure 2(a) extends existing visualizations, e.g., in [21, Figure 2], by adding material symmetries and motivates alternative parameterizations introduced in the next section.

2.4.I. Parameterizations of the orientation triangle. The classic parameterization of the orientation triangle in eigenvalues $\lambda_{1}$ and $\lambda_{2}$ is

$$
\mathbf{N}\left(\lambda_{1}, \lambda_{2}\right)=\lambda_{1} \mathbf{v}_{1} \otimes \mathbf{v}_{1}+\lambda_{2} \mathbf{v}_{2} \otimes \mathbf{v}_{2}+\left(1-\lambda_{1}-\lambda_{2}\right) \mathbf{v}_{3} \otimes \mathbf{v}_{3}
$$




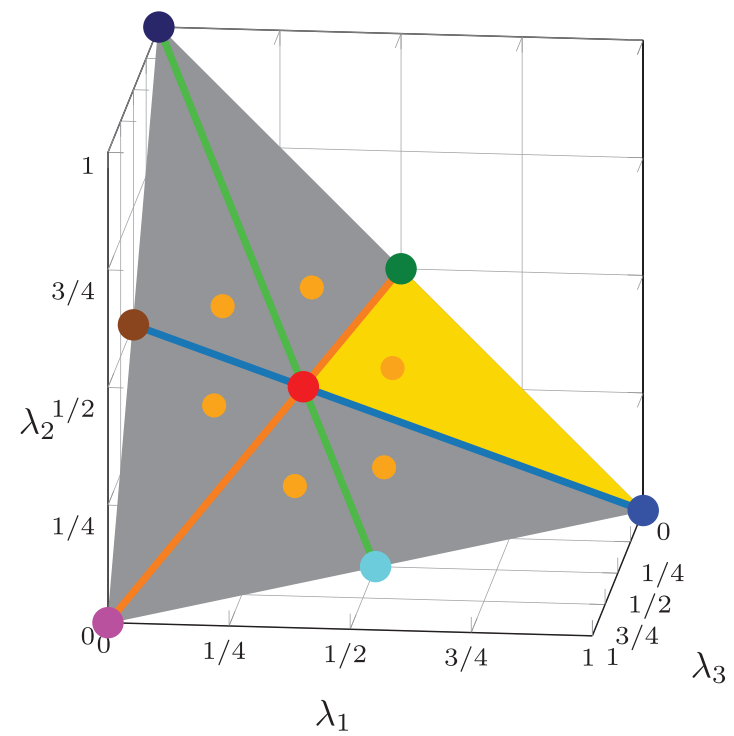

(a)

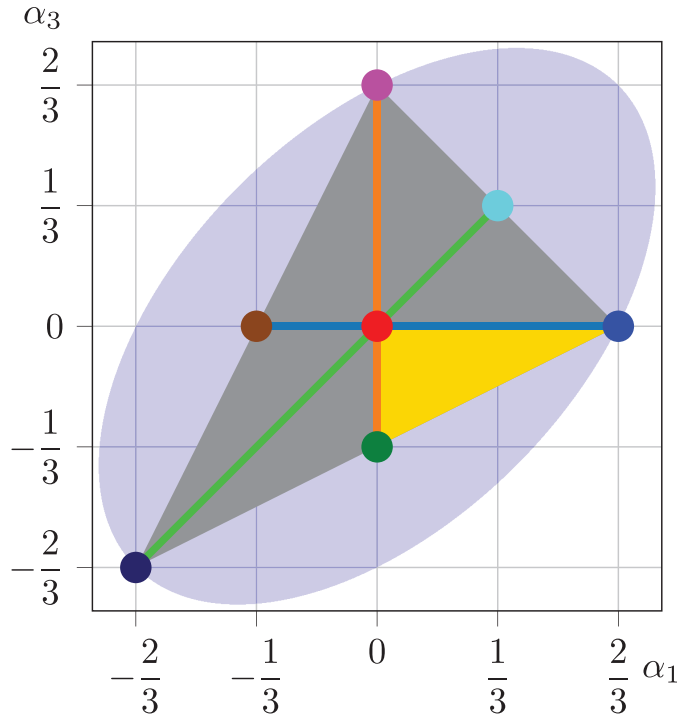

(b)

\begin{tabular}{|llll}
$|\mathbf{N}| \leq 1$ & plane & triangle & transv. iso. $\mathbf{v}_{1}$ \\
transv. iso. $\mathbf{v}_{3}$ & transv. iso. $\mathbf{v}_{2}$ & isotropic & unidirectional $\mathbf{v}_{1}$ \\
planar iso. $\mathbf{v}_{1}$ & unidirectional $\mathbf{v}_{2}$ & planar iso. $\mathbf{v}_{2}$ & unidirectional $\mathbf{v}_{3}$ \\
planar iso. $\mathbf{v}_{3}$ & perm $(1 / 2,1 / 3,1 / 6)$ & &
\end{tabular}

(c)

Figure 2. (a) Orientation plane, triangle, and selected orientation states in the space spanned by the eigenvalues of $\mathbf{N}$ (b) Plane, triangle, selected states, and states constrained by the norm of $\mathbf{N}$ in parameter space $\left(\alpha_{1}, \alpha_{3}\right)$ (c) Shared legend of (a) and (b).

with

$$
\frac{1}{3} \leq \lambda_{1} \leq 1 \quad \text { and } \quad \frac{1}{2}\left(1-\lambda_{1}\right) \leq \lambda_{2} \leq \min \left(\lambda_{1}, 1-\lambda_{1}\right) .
$$

Introducing two transversely isotropic deviatoric structure tensors

$$
\mathbf{F}^{\text {transv1 }}=\left[\begin{array}{ccc}
1 & 0 & 0 \\
& -1 / 2 & 0 \\
\text { sym } & & -1 / 2
\end{array}\right] \mathbf{v}_{i} \otimes \mathbf{v}_{j} \quad \text { and } \quad \mathbf{F}^{\text {transv3 }}=\left[\begin{array}{ccc}
-1 / 2 & 0 & 0 \\
& -1 / 2 & 0 \\
\text { sym } & & 1
\end{array}\right] \mathbf{v}_{i} \otimes \mathbf{v}_{j}
$$

enables an alternative parameterization of the orientation triangle by

$$
\mathbf{N}\left(\alpha_{1}, \alpha_{3}\right)=\mathbf{N}^{\text {iso }}+\alpha_{1} \mathbf{F}^{\text {transv1 }}+\alpha_{3} \mathbf{F}^{\text {transv3 }}
$$

with

$$
0 \leq \alpha_{1} \leq \frac{2}{3} \text { and } \frac{\alpha_{1}}{2}-\frac{1}{3} \leq \alpha_{3} \leq 0 .
$$

The parameterization in Equation (28) highlights the central role of the isotropic state, which is reached for $\alpha_{1}=\alpha_{3}=0$. Positive values of $\alpha_{1}$ lead to a deviation from the isotropic state towards the unidirectional state in direction $\mathbf{v}_{1}$. Deviation towards the planar isotropic state with principle axis $\mathbf{v}_{3}$, i.e., away from the unidirectional state in direction $\mathbf{v}_{3}$ is described by $\alpha_{3}$. Figure 2(b) shows the orientation triangle as part of the orientation plane in the parameter space $\left(\alpha_{1}, \alpha_{3}\right)$. The orientation plane itself is part of those pairs of $\left(\alpha_{1}, \alpha_{3}\right)$, which lead to orientations with norm less than one. If the separation into two transversely directions is not required, $\mathbf{F}^{\text {transv1 }}$ and $\mathbf{F}^{\text {transv3 }}$ can be combined to a generally orthotropic structure tensor of second order, leading to

$$
\mathbf{N}(\hat{a}, \hat{c})=\mathbf{N}^{\text {iso }}+\mathbf{F}^{\text {ortho }}(\hat{a}, \hat{c}) \quad \text { with } \quad \mathbf{F}^{\text {ortho }}(\hat{a}, \hat{c})=\left[\begin{array}{ccc}
\hat{a} & 0 & 0 \\
& -(\hat{a}+\hat{c}) & 0 \\
\operatorname{sym} & \hat{c}
\end{array}\right] \mathbf{v}_{i} \otimes \mathbf{v}_{j} .
$$


A parameterization reflecting the arbitrariness of the ordering convention on $\lambda_{i}$ and deploying barycentric coordinates is given as a function of three non-negative weights $0 \leq \hat{w}_{i}$ with $i \in[1,2,3]$ and $\hat{w}_{\Sigma}=\sum_{i=1}^{3} \hat{w}_{i}$ by

$$
\mathbf{N}\left(\hat{w}_{1}, \hat{w}_{2}, \hat{w}_{3}\right)=\mathbf{N}\left(\lambda_{1}=\frac{\hat{w}_{1}}{\hat{w}_{\Sigma}}, \lambda_{2}=\frac{\hat{w}_{2}}{\hat{w}_{\Sigma}}\right)
$$

requiring $\hat{w}_{\Sigma}>0$. See [21, Figure 2] for a visualization of this parameterization. Each parameterization given in Equations (25), (28), (30), and (31) in combination with a rotation, following Equation (22), can be used to represent all possible second-order orientation tensors. Table 2 in Appendix B lists parameters of special points in all parameterizations.

Parameterizations of all transversely isotropic $\mathbf{N}$, not being restricted to the standard orientation triangle, are given by

$$
\begin{aligned}
& \mathbf{N}^{\text {transv }}\left(\lambda_{1}\right)=\mathbf{N}\left(\lambda_{1}, \lambda_{2}=\left(1-\lambda_{1}\right) / 2\right) \\
& \mathbf{N}^{\text {transv }}\left(\alpha_{1}\right)=\mathbf{N}\left(\alpha_{1}, \alpha_{3}=0\right) .
\end{aligned}
$$

A parameterization of transversely isotropic $\mathbf{N}$ inside the orientation triangle requires a piece-wise definition due to the kink at the isotropic state leading to

$$
\mathbf{N}^{\text {transv }}\left(\lambda_{2}\right)= \begin{cases}\left(1-2 \lambda_{2}\right) \mathbf{v}_{1} \otimes \mathbf{v}_{1}+\lambda_{2} \mathbf{v}_{2} \otimes \mathbf{v}_{2}+\lambda_{2} \mathbf{v}_{3} \otimes \mathbf{v}_{3} & \text { if } 0 \leq \lambda_{2} \leq \frac{1}{3} \\ \lambda_{2} \mathbf{v}_{1} \otimes \mathbf{v}_{1}+\lambda_{2} \mathbf{v}_{2} \otimes \mathbf{v}_{2}+\left(1-2 \lambda_{2}\right) \mathbf{v}_{3} \otimes \mathbf{v}_{3} & \text { if } \frac{1}{3}<\lambda_{2} \leq \frac{1}{2}\end{cases}
$$

Planar second-order orientation tensors inside the orientation triangle can be parameterized by, e.g.,

$$
\mathbf{N}^{\text {planar }}\left(\alpha_{1}\right)=\mathbf{N}\left(\alpha_{1}, \alpha_{3}=\frac{\alpha_{1}}{2}-\frac{1}{3}\right)=\left(\frac{1}{2}+\frac{3}{4} \alpha_{1}\right) \mathbf{v}_{1} \otimes \mathbf{v}_{1}+\left(\frac{1}{2}-\frac{3}{4} \alpha_{1}\right) \mathbf{v}_{2} \otimes \mathbf{v}_{2}
$$

with $0 \leq \alpha_{1} \leq 2 / 3$.

\subsection{Variety of fourth-order orientation tensors}

Equation (10) implies that $\mathbb{N}$ is completely (or totally) symmetric, i.e., any permutation of the indices

$$
N_{i j k l}^{(4)}=N_{\text {perm }(i j k l)}^{(4)}
$$

holds for a representation with tensor coefficients $\mathbb{N}=N_{i j k l}^{(4)} \mathbf{e}_{i} \otimes \mathbf{e}_{j} \otimes \mathbf{e}_{k} \otimes \mathbf{e}_{l}$. Complete (index) symmetry reduces the number of independent components of a generic fourth-order tensor in three dimensions from $81=3^{4}$ to 15 , as there are $15=\left(\begin{array}{c}n+k-1 \\ k\end{array}\right)$ unordered ways of choosing a combination of $k=4$ elements from a set of $n=3$ elements. Mandel notation, introduced in [23] and also known as normalized Voigt notation $[31,35]$ enables compact two-dimensional representations of fourth-order tensors with at least minor symmetry. A fourth-order tensor $\mathbb{A}=A_{i j k l} \mathbf{e}_{i} \otimes \mathbf{e}_{j} \otimes \mathbf{e}_{k} \otimes \mathbf{e}_{l}$ is minor symmetric if it has both minor symmetries, i.e., $A_{i j k l}=A_{j i k l}=A_{i j l k}$ holds. Introducing base tensors in an arbitrary Cartesian basis $\left\{\mathbf{e}_{i}\right\}$ by

$$
\begin{array}{ll}
\boldsymbol{B}_{1}=\boldsymbol{e}_{1} \otimes \boldsymbol{e}_{1}, & \boldsymbol{B}_{4}=\frac{\sqrt{2}}{2}\left(\boldsymbol{e}_{2} \otimes \boldsymbol{e}_{3}+\boldsymbol{e}_{3} \otimes \boldsymbol{e}_{2}\right), \\
\boldsymbol{B}_{2}=\boldsymbol{e}_{2} \otimes \boldsymbol{e}_{2}, & \boldsymbol{B}_{5}=\frac{\sqrt{2}}{2}\left(\boldsymbol{e}_{1} \otimes \boldsymbol{e}_{3}+\boldsymbol{e}_{3} \otimes \boldsymbol{e}_{1}\right), \\
\boldsymbol{B}_{3}=\boldsymbol{e}_{3} \otimes \boldsymbol{e}_{3}, & \boldsymbol{B}_{6}=\frac{\sqrt{2}}{2}\left(\boldsymbol{e}_{2} \otimes \boldsymbol{e}_{1}+\boldsymbol{e}_{1} \otimes \boldsymbol{e}_{2}\right),
\end{array}
$$


a minor symmetric tensor $\mathbb{A}$ is represented by a six-by-six matrix of coefficients $A_{\xi \zeta}$

$$
\begin{aligned}
\mathbb{A} & =A_{i j k l} \mathbf{e}_{i} \otimes \mathbf{e}_{j} \otimes \mathbf{e}_{k} \otimes \mathbf{e}_{l}=A_{\xi \zeta} \mathbf{B}_{\xi} \otimes \mathbf{B}_{\zeta} \\
& =\left[\begin{array}{llllll}
A_{1111} & A_{1122} & A_{1133} & \sqrt{2} A_{1123} & \sqrt{2} A_{1113} & \sqrt{2} A_{1112} \\
A_{2211} & A_{2222} & A_{2233} & \sqrt{2} A_{2223} & \sqrt{2} A_{2213} & \sqrt{2} A_{2212} \\
A_{3311} & A_{3322} & A_{333} & \sqrt{2} A_{3323} & \sqrt{2} A_{3313} & \sqrt{2} A_{3312} \\
\sqrt{2} A_{2311} & \sqrt{2} A_{2322} & \sqrt{2} A_{2333} & 2 A_{2323} & 2 A_{2313} & 2 A_{2312} \\
\sqrt{2} A_{1311} & \sqrt{2} A_{1322} & \sqrt{2} A_{1333} & 2 A_{1323} & 2 A_{1313} & 2 A_{1312} \\
\sqrt{2} A_{1211} & \sqrt{2} A_{1222} & \sqrt{2} A_{1233} & 2 A_{1223} & 2 A_{1213} & 2 A_{1212}
\end{array}\right] \mathbf{B}_{\xi} \otimes \mathbf{B}_{\zeta}
\end{aligned}
$$

with $\xi$ and $\zeta$ summing from 1 to 6 . A Hooke tensor $\mathbb{B}$ is minor and major symmetric, i.e., in addition to the minor symmetry condition it holds that $B_{i j k l}=B_{k l i j}$. The coefficient matrix of a Hooke tensor

$$
\mathbb{B}=\left[\begin{array}{llllll}
B_{11} & B_{12} & B_{13} & \sqrt{2} B_{14} & \sqrt{2} B_{15} & \sqrt{2} B_{16} \\
B_{12} & B_{22} & B_{23} & \sqrt{2} B_{24} & \sqrt{2} B_{25} & \sqrt{2} B_{26} \\
B_{13} & B_{23} & B_{33} & \sqrt{2} B_{34} & \sqrt{2} B_{35} & \sqrt{2} B_{36} \\
\sqrt{2} B_{14} & \sqrt{2} B_{24} & \sqrt{2} B_{34} & 2 B_{44} & 2 B_{45} & 2 B_{46} \\
\sqrt{2} B_{15} & \sqrt{2} B_{25} & \sqrt{2} B_{35} & 2 B_{45} & 2 B_{55} & 2 B_{56} \\
\sqrt{2} B_{16} & \sqrt{2} B_{26} & \sqrt{2} B_{36} & 2 B_{46} & 2 B_{56} & 2 B_{66}
\end{array}\right] \mathbf{B}_{\xi} \otimes \mathbf{B}_{\zeta}
$$

is symmetric and contains 21 independent parameters. Complete index symmetry of $\mathbb{N}$ implies

$$
\mathbb{N}=\left[\begin{array}{ccc|ccc}
N_{11}^{(4)} & N_{12}^{(4)} & N_{13}^{(4)} & \sqrt{2} N_{14}^{(4)} & \sqrt{2} N_{15}^{(4)} & \sqrt{2} N_{16}^{(4)} \\
& N_{22}^{(4)} & N_{23}^{(4)} & \sqrt{2} N_{24}^{(4)} & \sqrt{2} N_{25}^{(4)} & \sqrt{2} N_{26}^{(4)} \\
& & N_{33}^{(4)} & \sqrt{2} N_{34}^{(4)} & \sqrt{2} N_{35}^{(4)} & \sqrt{2} N_{36}^{(4)} \\
\hline & & 2 N_{23}^{(4)} & 2 N_{36}^{(4)} & 2 N_{25}^{(4)} \\
\text { major symmetric } & & 2 N_{13}^{(4)} & 2 N_{14}^{(4)} \\
& & & & 2 N_{12}^{(4)}
\end{array}\right] \mathbf{B}_{\xi} \otimes \mathbf{B}_{\zeta} .
$$

In Equation (40) indices of redundant tensor coefficients are colored. The redundancy implies that six coefficients in the upper left quadrant and nine coefficients in the upper right quadrant of the coefficients in Mandel notation define a completely symmetric tensor. This motivates a shorthand notation "completely symmetric," which to the best of the authors' knowledge has not been used in literature so far, see, e.g., Equation (53). As $\mathbb{N}$ contains $\mathbf{N}$, the constraint on the trace of $\mathbf{N}$ in Equation (12) reduces the number of independent components of $\mathbb{N}$ by one to 14. In literature erroneous implications of Equation (12) on the number of independent components of $\mathbb{N}$ are found, see, e.g., [36]. Expressing $\mathbf{N}$ by contraction of $\mathbb{N}$, i.e.,

$$
\mathbf{N}=\left[\begin{array}{ccc}
N_{11}^{(4)}+N_{12}^{(4)}+N_{13}^{(4)} & N_{16}^{(4)}+N_{26}^{(4)}+N_{36}^{(4)} & N_{15}^{(4)}+N_{25}^{(4)}+N_{35}^{(4)} \\
N_{16}^{(4)}+N_{26}^{(4)}+N_{36}^{(4)} & N_{12}^{(4)}+N_{22}^{(4)}+N_{23}^{(4)} & N_{14}^{(4)}+N_{24}^{(4)}+N_{34}^{(4)} \\
N_{15}^{(4)}+N_{25}^{(4)}+N_{35}^{(4)} & N_{14}^{(4)}+N_{24}^{(4)}+N_{34}^{(4)} & N_{13}^{(4)}+N_{23}^{(4)}+N_{33}^{(4)}
\end{array}\right] \boldsymbol{e}_{i} \otimes \boldsymbol{e}_{j}
$$

reveals the implication of Equation (12) on $\mathbb{N}$ to be $N_{11}^{(4)}+N_{22}^{(4)}+N_{33}^{(4)}+2 N_{12}^{(4)}+2 N_{13}^{(4)}+2 N_{23}^{(4)}=1$.

2.5.I. Harmonic decomposition. Linear invariant decompositions, including the classic harmonic decomposition, are frequently used on Hooke tensors. The fourth-order orientation tensor $\mathbb{N}$ is a Hooke tensor and has additional index symmetry. Therefore, linear invariant decompositions can be applied to $\mathbb{N}$ to study its structure. Literature on invariant decompositions of fourth-order tensors is extensive, see, e.g., [30, 37-41] or those focusing on Hooke tensors and addressing an engineering audience [42-44].

Following [42], a Hooke tensor $\mathbb{H}$ with 21 independent components can be split into five parts $\left(K, G, \mathbf{H}_{1}, \mathbf{H}_{2}, \operatorname{dev}(\mathbb{H})\right)$ leading to

$$
\mathbb{H}=3 K \mathbb{P}_{1}+2 G \mathbb{P}_{2}+\operatorname{sym}_{1}\left(\mathbf{I}_{2} \otimes \mathbf{H}_{1}+\mathbf{H}_{1} \otimes \mathbf{I}_{2}\right)+\operatorname{sym}_{2}\left(\mathbf{I}_{2} \otimes \mathbf{H}_{2}+\mathbf{H}_{2} \otimes \mathbf{I}_{2}\right)+\operatorname{dev}(\mathbb{H})
$$


with isotropic projectors $\mathbb{P}_{1}$ and $\mathbb{P}_{2}$, two symmetrization operators $\operatorname{sym}_{1}(\cdot), \operatorname{sym}_{2}(\cdot)$ and the deviatoric operator $\operatorname{dev}(\cdot)$. The symmetrization operators have to follow a special structure which is described in [42]. Two common choices of the symmetrization operators are used in the following. The numbers of independent parameters of the five parts $\left(K, G, \mathbf{H}_{1}, \mathbf{H}_{2}\right.$, dev $\left.(\mathbb{H})\right)$ are $(1,1,5,5,9)$ with the scalar compression modulus $K$ and shear modulus $G$. The isotropic parts are obtained by projections, i.e.,

$$
3 K=\mathbb{P}_{1} \cdot \mathbb{H}, \quad 2 G=\frac{1}{\left\|\mathbb{P}_{2}\right\|^{2}} \mathbb{P}_{2} \cdot \mathbb{H}=\frac{1}{5} \mathbb{P}_{2} \cdot \mathbb{H} .
$$

The parts $\mathbf{H}_{1}$ and $\mathbf{H}_{2}$ depend on the choice of the operators $\operatorname{sym}_{1}(\cdot)$ and $\operatorname{sym}_{2}(\cdot)$ but for any choice are only functions of the dilatational modulus $\mathbf{C}$ and the Voigt tensor $\mathbf{V}$ which are given in index notation by

$$
C_{i j}=H_{i j k k} \quad \text { and } \quad V_{i j}=H_{i k j k}
$$

with $\mathbf{C}=C_{i j} \mathbf{e}_{i} \otimes \mathbf{e}_{j}, \mathbf{V}=V_{i j} \mathbf{e}_{i} \otimes \mathbf{e}_{j}$ and $\mathbb{H}=H_{i j k l} \mathbf{e}_{i} \otimes \mathbf{e}_{j} \otimes \mathbf{e}_{k} \otimes \mathbf{e}_{l}$. The classic harmonic decomposition, e.g., used in [31], is obtained for $\operatorname{sym}_{1}(\cdot)$ being the identity and $\operatorname{sym}_{2}(\cdot)$ symmetrizing the only remaining index asymmetry of Hooke tensors, leading to

$$
\begin{aligned}
\mathbf{H}_{1}^{\text {classic }} & =\frac{1}{7}(5 \operatorname{dev}(\mathbf{C})-4 \operatorname{dev}(\mathbf{V})) \\
\mathbf{H}_{2}^{\text {classic }} & =\frac{1}{7}(3 \operatorname{dev}(\mathbf{C})-2 \operatorname{dev}(\mathbf{V})) .
\end{aligned}
$$

Applying the classic harmonic decomposition to $\mathbb{N}$ reveals that the dilatational modulus and the Voigt modulus of $\mathbb{N}$ coincide and both are $\mathbf{N}$. Following [45], a Hooke tensor whose dilatational modulus and Voigt tensor coincide is said to fulfill the Cauchy relations. Any completely symmetric tensor and, thus, also any orientation tensor fulfills the Cauchy relations. Referencing [46], Cowin [45] states about the Cauchy relations

[...] the relations do not hold for most elastic materials, but only for materials which can be described as having central-force laws operating between points of a simple lattice, [...]

This observation fits the model of fibers pointing towards the origin of a unit sphere. A stiffness which is linear in the orientation tensors does not have to fulfill the Cauchy relations as its deviatoric parts $\left(\mathbf{H}_{1}\right.$ and $\mathbf{H}_{2}$ in Equation (42)) might be multiples of $\mathbf{N}$ and, therefore, do not coincide.

Motivated by their orthogonality, Rychlewski [42] proposed an alternative pair of symmetrization operators $\operatorname{sym}_{1}(\cdot)=\operatorname{sym}(\cdot)$ and $\operatorname{sym}_{2}(\cdot)=\mathbb{I}^{S}-\operatorname{sym}_{1}(\cdot)$ focusing on the index symmetry with the identity on symmetric fourth-order tensors $\mathbb{I}^{S}$. This choice leads to the corresponding parts

$$
\begin{aligned}
& \mathbf{H}_{1}^{\text {index }}=\frac{1}{7}(\operatorname{dev}(\mathbf{C})+2 \operatorname{dev}(\mathbf{V})) \\
& \mathbf{H}_{2}^{\text {index }}=\operatorname{dev}(\mathbf{C})-\operatorname{dev}(\mathbf{V}) .
\end{aligned}
$$

As a consequence, $\mathbb{N}$ can be decomposed into

$$
\begin{aligned}
\mathbb{N} & =3 \hat{K} \mathbb{P}_{1}+2 \hat{G} \mathbb{P}_{2}+\operatorname{sym}\left(\mathbf{I}_{2} \otimes \frac{3}{7} \operatorname{dev}(\mathbf{N})+\frac{3}{7} \operatorname{dev}(\mathbf{N}) \otimes \mathbf{I}_{2}\right)+\operatorname{dev}(\mathbb{N}) \\
& =3 \hat{K} \mathbb{P}_{1}+2 \hat{G} \mathbb{P}_{2}+2 \operatorname{sym}\left(\frac{3}{7} \operatorname{dev}(\mathbf{N}) \otimes \mathbf{I}_{2}\right)+\operatorname{dev}(\mathbb{N}) \\
& =3 \hat{K} \mathbb{P}_{1}+2 \hat{G} \mathbb{P}_{2}+\frac{6}{7} \operatorname{sym}\left(\operatorname{dev}(\mathbf{N}) \otimes \mathbf{I}_{2}\right)+\operatorname{dev}(\mathbb{N})
\end{aligned}
$$

Due to the isotropy of the isotropic projectors, the linearity of the scalar product and the normalization of $\psi(\mathbf{n})$, the projection of $\mathbb{N}$ on either of the isotropic projectors is

$$
\mathbb{P}_{i} \cdot \mathbb{N}=\mathbb{P}_{i} \cdot \int_{\mathcal{S}^{2}} \psi(\mathbf{n}) \mathbf{n}^{\otimes 4} \mathrm{~d} n=\mathbb{P}_{i} \cdot \mathbf{n}^{\otimes 4} \quad \text { with } i \in[1,2]
$$


Consequently, the isotropic part of $\mathbb{N}$ can be determined analyzing the fourth-order moment $\mathbf{n}^{\otimes 4}$ which is given in Mandel notation by

$$
\mathbf{n}^{\otimes 4}=\left[\begin{array}{ccc|ccc}
n_{1}^{4} & n_{1}^{2} n_{2}^{2} & n_{1}^{2} n_{3}^{2} & \sqrt{2} n_{1}^{2} n_{2} n_{3} & \sqrt{2} n_{1}^{3} n_{3} & \sqrt{2} n_{1}^{3} n_{2} \\
& n_{2}^{4} & n_{2}^{2} n_{3}^{2} & \sqrt{2} n_{2}^{3} n_{3} & \sqrt{2} n_{1} n_{2}^{2} n_{3} & \sqrt{2} n_{1} n_{2}^{3} \\
& & n_{3}^{4} & \sqrt{2} n_{2} n_{3}^{3} & \sqrt{2} n_{1} n_{3}^{3} & \sqrt{2} n_{1} n_{2} n_{3}^{2} \\
\hline \multirow{2}{*}{\text { completely }} & & & \text { symmetric } &
\end{array}\right] \mathbf{B}_{\xi} \otimes \mathbf{B}_{\zeta}
$$

leading to

$$
\begin{aligned}
3 \hat{K}=\mathbb{P}_{1} \cdot \mathbf{n}^{\otimes 4} & =\frac{1}{3}\left[\begin{array}{cccccc}
1 & 1 & 1 & 0 & 0 & 0 \\
1 & 1 & 1 & 0 & 0 & 0 \\
1 & 1 & 1 & 0 & 0 & 0 \\
0 & 0 & 0 & 0 & 0 & 0 \\
0 & 0 & 0 & 0 & 0 & 0 \\
0 & 0 & 0 & 0 & 0 & 0
\end{array}\right] \mathbf{B}_{\xi} \otimes \mathbf{B}_{\zeta} \cdot\left(\mathbf{n}^{\otimes 4}\right) \\
& =\frac{1}{3}\left(n_{1}^{4}+2 n_{1}^{2} n_{2}^{2}+2 n_{1}^{2} n_{3}^{2}+n_{2}^{4}+2 n_{2}^{2} n_{3}^{2}+n_{3}^{4}\right)=\frac{1}{3}
\end{aligned}
$$

and

$$
2 \hat{G}=\frac{\mathbb{P}_{2}}{\left\|\mathbb{P}_{2}\right\|^{2}} \cdot \mathbf{n}^{\otimes 4}=\frac{1}{15}\left[\begin{array}{cccccc}
2 & -1 & -1 & 0 & 0 & 0 \\
-1 & 2 & -1 & 0 & 0 & 0 \\
-1 & -1 & 2 & 0 & 0 & 0 \\
0 & 0 & 0 & 3 & 0 & 0 \\
0 & 0 & 0 & 0 & 3 & 0 \\
0 & 0 & 0 & 0 & 0 & 3
\end{array}\right] \mathbf{B}_{\xi} \otimes \mathbf{B}_{\zeta} \cdot\left(\mathbf{n}^{\otimes 4}\right)=\frac{2}{15} .
$$

Thus, the isotropic part of any fourth-order fiber orientation tensor is fixed due to the normalization of both $\psi(\mathbf{n})$ and $\mathbf{n}$ and is given by

$$
\mathbb{N}^{\text {iso }}=3 \hat{K} \mathbb{P}_{1}+2 \hat{G} \mathbb{P}_{2}=\frac{1}{15}\left[\begin{array}{cccccc}
3 & 1 & 1 & 0 & 0 & 0 \\
1 & 3 & 1 & 0 & 0 & 0 \\
1 & 1 & 3 & 0 & 0 & 0 \\
0 & 0 & 0 & 2 & 0 & 0 \\
0 & 0 & 0 & 0 & 2 & 0 \\
0 & 0 & 0 & 0 & 0 & 2
\end{array}\right] \mathbf{B}_{\xi} \otimes \mathbf{B}_{\zeta}=\frac{7}{35} \operatorname{sym}\left(\mathbf{I}_{2} \otimes \mathbf{I}_{2}\right)
$$

leading to a compact representation of Equation (51) by

$$
\mathbb{N}=\mathbb{N}^{\text {iso }}+\frac{6}{7} \operatorname{sym}\left(\operatorname{dev}(\mathbf{N}) \otimes \mathbf{I}_{2}\right)+\operatorname{dev}(\mathbb{N}) .
$$

This representation emphasizes isotropy as the one element of directional measures. Equation (57) reveals that in contrast to the well-known harmonic decomposition of Hooke tensors, the harmonic decomposition of fourthorder orientation tensors only contains one irreducible subspace of second-order and the isotropic subspace degenerates from two scalars to a constant value. Coincidence of Equations (57) and (19) is given by

$$
\operatorname{sym}\left(\mathbf{I}_{2} \otimes \operatorname{dev}(\mathbf{N})\right)=\operatorname{sym}\left(\mathbf{I}_{2} \otimes \mathbf{N}\right)-\frac{1}{3} \operatorname{sym}\left(\mathbf{I}_{2} \otimes \mathbf{I}_{2}\right) .
$$

Interpretation of $\mathbb{N}$ as a Hooke tensor and applying the harmonic decomposition translates the reasoning of [1] into the language of continuum mechanics. Here $\mathbb{N}$ can be parameterized by two deviators, one being of second and the other of fourth order. Both represent the deviation from the isotropic state. Five of the 14 independent parameters of $\mathbb{N}$ define the second-order deviator and nine define the fourth-order deviator. Three independent parameters of the second-order deviator define an orientation coordinate system in which both deviators have simplified representations (see the structure tensor in Equation (30) and the following section). 
Table I. Implications of symmetry classes of the ODF $\psi$ on the second- and fourth-order orientation tensors

\begin{tabular}{lll}
\hline & Material symmetry reflected by & \\
Symmetry class of $\psi$ & $\mathbb{N}$ & $\mathbf{N}$ \\
\hline \hline $\begin{array}{l}\text { isotropic } \\
\text { cubic }\end{array}$ & isotropic & isotropic \\
cubic & isotropic \\
\hline transv. iso. & transv. iso. & transv. iso. \\
tetragonal & tetragonal & transv. iso. \\
trigonal & trigonal & transv. iso. \\
\hline $\begin{array}{l}\text { orthotropic } \\
\text { monoclinic }\end{array}$ & orthotropic & orthotropic \\
triclinic & monoclinic & orthotropic \\
\hline
\end{tabular}

2.5.2. Parameterizations and admissible parameter ranges of $\mathbb{N}$. The space of fourth-order orientation tensors $\mathbb{N}$, which fulfill the algebraic constraints imposed by Equation (8) and which can be derived from an ODF, remains to be identified. The variety of $\mathbf{N}$ is known from Section 2.4. Therefore, Equation (57) reveals that the analysis of the variety of $\mathbb{N}$ is reduced to the identification of a fourth-order deviator $\operatorname{dev}(\mathbb{N})$. This fourth-order deviator reflects the material symmetry of the ODF and, consequently, also of $\mathbf{N}$ and $\mathbb{N}$. As a consequence, special representations of the fourth-order deviator in the orientation coordinate system exist. Knowledge on material symmetries of Hooke tensors is applied to identify admissible ranges of the fourth-order deviator. The most general fourth-order orientation tensor has the structure

$$
\mathbb{N}\left(\mathbf{N}, d_{1}, \ldots, d_{9}\right)=\mathbb{N}^{\text {iso }}+\frac{6}{7} \operatorname{sym}\left(\operatorname{dev}(\mathbf{N}) \otimes \mathbf{I}_{2}\right)+\mathbb{F}^{\text {tricl }}\left(d_{1}, \ldots, d_{9}\right)
$$

with a triclinic deviatoric structure tensor

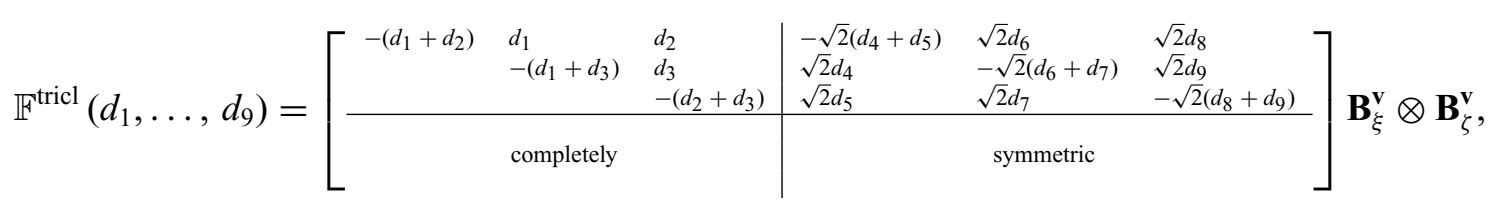

which is a function of nine scalar parameters. The Mandel basis $\mathbf{B}_{\xi}^{\mathbf{v}} \otimes \mathbf{B}_{\zeta}^{\mathbf{v}}$ is spanned in the orientation coordinate system, for example, the first definition in Equation (37) becomes $\boldsymbol{B}_{1}=\mathbf{v}_{1} \otimes \mathbf{v}_{1}$. Without loss of generality, any orthotropic parameterization of N, e.g., Equation (33), can be combined with Equation (60) yielding a set of parameters, e.g., $\left(\alpha_{1}, \alpha_{3}, d_{1}, \ldots, d_{9}\right)$. If the ODF has a material symmetry, this symmetry also applies to the orientation tensors and reduces the number of independent parameters, see, e.g., the implications of transverse isotropy on the parameterization of $\mathbf{N}$ in Equation (33). For a given material symmetry class, the number of independent parameters is fixed. All eight material symmetries imply different constraints on $\mathbb{N}$. However, e.g., triclinic, monoclinic and orthotropic symmetry of $\psi$ lead to identical constraints on $\mathbf{N}$. In other words, triclinic, monoclinic, and orthotropic symmetric second-order tensors coincide and are usually referred to as orthotropic (see Appendix A). Implications of the symmetry of the ODF on the symmetry of $\mathbf{N}$ and $\mathbb{N}$ are listed in Table 1.

The hierarchy of material symmetries including visualizations of symmetry planes is given in [32, Figure 4]. As a consequence, index and material symmetry constrain the space of $\mathbb{N}$. The question on the variety of fiber orientation tensors is reduced to the determination of admissible parameter combinations. The flexible parameterization of fourth-order fiber orientation tensors in Equation (59) is new, highlights the isotropic state, and can be easily adapted to stronger material symmetries.

The remaining algebraic constraint on $\mathbb{N}$ is positive semi-definiteness. Positive semi-definiteness implies that

$$
0 \leq \mathbb{N} \cdot(\mathbf{A} \otimes \mathbf{A})
$$

holds for any second-order tensor A. Without loss of generality, A can be restricted to symmetric second-order tensors as $\mathbb{N}$ is symmetric and skew, and symmetric parts are orthogonal. As a consequence, Equation (61) can 
be written in Mandel notation stating a root finding problem of the characteristic polynomial of $\mathbb{N}$ of degree six. Here $\mathbb{N}$ is positive semi-definite if all of its eigenvalues $\Lambda_{i}$ are non-negative, i.e.,

$$
0 \leq \Lambda_{i} \quad \text { for } i \in[1, \ldots, 6]
$$

holds. Explicit representations of the eigenvalues of $\mathbb{N}$ are given for a limited number of material symmetries in [35]. Eigenvalues of fourth-order tensors can not be identified from coefficient matrices in Voigt notation. Mandel notation has to be used [23, 35, 44].

2.5.3. Transversely isotropic case. A transversely isotropic harmonic, i.e., completely symmetric and completely traceless, tensor of fourth order with principle axis in direction $\mathbf{e}_{1}$ is a multiple of the structure tensor

$$
\mathbb{F}^{\text {transv1 }}=\left[\begin{array}{ccc|ccc}
8 & -4 & -4 & 0 & 0 & 0 \\
3 & 1 & 0 & 0 & 0 \\
& 3 & 0 & 0 & 0 \\
\hline \text { completely } & \text { symmetric }
\end{array}\right] \mathbf{B}_{\xi} \otimes \mathbf{B}_{\zeta}=\mathbb{F}^{\text {tricl }}(-4,-4,1,0,0,0,0,0,0),
$$

see, e.g., [29, (75)], [47, A.3], or [5, (11)] and can be expressed in terms of the triclinic structure tensor defined in Equation (60). As a consequence, transversely isotropic fourth-order orientation tensors are parameterized in the orientation coordinate system by

$$
\begin{aligned}
\mathbb{N}^{\text {transv }}(\alpha, \rho) & =\mathbb{N}^{\text {iso }}+\alpha \frac{6}{7} \operatorname{sym}\left(\mathbf{F}^{\text {transv1 }} \otimes \mathbf{I}_{2}\right)+\rho \mathbb{F}^{\text {transv1 }} \\
& =\mathbb{N}^{\text {iso }}+\left[\begin{array}{lll|lll}
6 \alpha / 7+8 \rho & \alpha / 14-4 \rho & \alpha / 14-4 \rho & 0 & 0 & 0 \\
& -3 \alpha / 7+3 \rho & -\alpha / 7+\rho & 0 & 0 & 0 \\
& & -3 \alpha / 7+3 \rho & 0 & 0 & 0 \\
\hline & \text { completely } & \text { symmetric }
\end{array}\right] \mathbf{B}_{\xi}^{\mathbf{v}} \otimes \mathbf{B}_{\zeta}^{\mathbf{v}} .
\end{aligned}
$$

The choice of the principle axis only effects the mapping $\mathbf{Q}$, defined in Equation (22), and parameterizations around $\mathbf{v}_{2}$ or $\mathbf{v}_{3}$ are equivalent, see, e.g., [5]. Demanding positive eigenvalues leads to the set of admissible transversely isotropic fourth-order orientation tensors

$$
\mathcal{N}^{\text {transv }}=\left\{\mathbb{N}^{\text {transv }}(\alpha, \rho) \mid-\frac{1}{3} \leq \alpha \leq \frac{2}{3}, \frac{1}{8} \alpha^{2}+\frac{1}{42} \alpha-\frac{1}{90} \leq \rho \leq \frac{1}{56} \alpha+\frac{1}{60}\right\}
$$

given, e.g., in $[5,13]$. The set $\mathcal{N}^{\text {transv }}$ combined with the parameterization in Equation (64) represents the variety of transversely isotropic fourth-order orientation tensors [5].

An alternative derivation of $\mathcal{N}^{\text {transv }}$ is given by [13, Equation (54)] deploying the Schwarz inequality on directional cosines to identify a relationship between $\mathbf{N}$ and $\mathbb{N}$. This relationship implies constraints on the coefficients of $\mathbb{N}$, which are reformulated in the notation of this work as

$$
\left(N_{k k}^{(2)} \mathbf{v}_{k} \otimes \mathbf{v}_{k}\right)^{2} \leq N_{k k}^{(4)} \mathbf{B}_{k}^{\mathbf{v}} \otimes \mathbf{B}_{k}^{\mathbf{v}} \leq N_{k k}^{(2)} \mathbf{v}_{k} \otimes \mathbf{v}_{k}
$$

for $k \in[1,2,3]$ without summation convention. To be precise, the set

$$
\mathcal{N}_{\text {cosines }}^{\text {transv }}=\left\{\mathbb{N}^{\text {transv }} \mid\left(N_{k k}^{(2)} \mathbf{v}_{k} \otimes \mathbf{v}_{k}\right)^{2} \leq N_{k k}^{(4)} \mathbf{B}_{k}^{\mathbf{v}} \otimes \mathbf{B}_{k}^{\mathbf{v}} \leq N_{k k}^{(2)} \mathbf{v}_{k} \otimes \mathbf{v}_{k}\right\}
$$

is identical to the set $\mathcal{N}^{\text {transv }}$. However, for weaker symmetries, positive definiteness is a stronger constraint than the conditions in Equation (66). Comparison of second- and fourth-order moment tensors (see, e.g., Equation (53)) motivates the reasoning behind Equation (66). In order to frame the implications of positive 


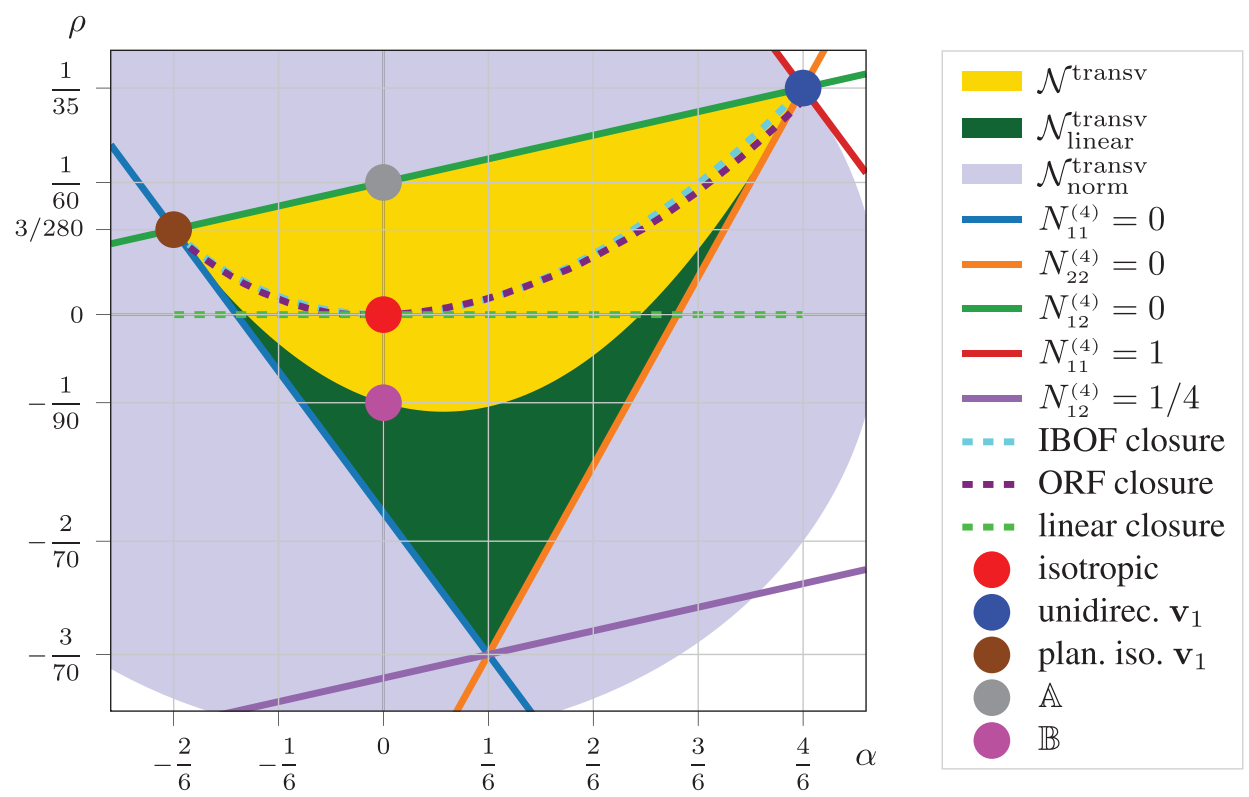

Figure 3. Transversely isotropic fourth-order orientation states following [13].

semi-definiteness, Figure 3 contains additional subsets, lines and special points discussed in the following. Interpretation of $\mathbb{N}$ as weighted summation of moment tensors leads to coefficient-wise constraints on $\mathbb{N}$ (see Appendix C). The set $\mathcal{N}_{\text {linear }}^{\text {trans }}$, defined in Equation (104), fulfills these coefficient-wise constraints. The set of transversely isotropic orientation states bounded by the norm of a singular orientation is denoted by

$$
\mathcal{N}_{\text {norm }}^{\text {transv }}=\left\{\mathbb{N}^{\text {transv }} \mid 0 \leq\|\mathbb{N}\|_{\text {Frobenius }} \leq 1\right\} .
$$

Figure 3 visualizes the relation $\mathcal{N}^{\text {transv }} \subset \mathcal{N}_{\text {linear }}^{\text {transv }} \subset \mathcal{N}_{\text {norm }}^{\text {transv }}$. Special orientation states, such as the planar isotropic and unidirectional state, are marked in Figure 3 for reference. The isotropic state $\mathbb{N}^{\text {iso }}$, defined in Equation (56), is located at $\alpha=\rho=0$ and is part of a straight vertical line with $\alpha=0$ which contains orientation states with isotropic second-order tensor. Therefore, the set of transversely isotropic orientation states with isotropic second-order orientation tensor is a one-dimensional subspace of $\mathcal{N}^{\text {transv }}$ with limiting orientation states visualized in Figure 3 and given by

$$
\begin{aligned}
\mathbb{A} & =\mathbb{N}^{\text {transv }}(\alpha=0, \rho=1 / 60) \\
& =\left[\begin{array}{lll|lll}
1 / 3 & 0 & 0 & 0 & 0 & 0 \\
& 1 / 4 & 1 / 12 & 0 & 0 & 0 \\
& 1 / 4 & 0 & 0 & 0 \\
\hline & \text { completely } & \text { symmetric }
\end{array}\right] \mathbf{B}_{\xi}^{\mathbf{v}} \otimes \mathbf{B}_{\zeta}^{\mathbf{v}}
\end{aligned}
$$

and

$$
\begin{aligned}
& \mathbb{B}=\mathbb{N}^{\text {transv }}(\alpha=0, \rho=-1 / 90) \\
& =\left[\begin{array}{ccc|ccc}
1 / 9 & 1 / 9 & 1 / 9 & 0 & 0 & 0 \\
1 / 6 & 1 / 18 & 0 & 0 & 0 \\
& 1 / 6 & 0 & 0 & 0 \\
\hline & \text { completely } & \text { symmetric }
\end{array}\right] \mathbf{B}_{\xi}^{\mathbf{v}} \otimes \mathbf{B}_{\zeta}^{\mathbf{v}} .
\end{aligned}
$$

The states $\mathbb{A}$ and $\mathbb{B}$ cannot be distinguished by means of second-order orientation tensors as

$$
\mathbb{A}\left[\mathbf{I}_{2}\right]=\mathbb{B}\left[\mathbf{I}_{2}\right]=\mathbf{N}^{\text {iso }}
$$




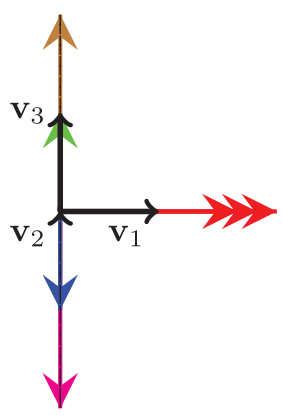

(a)

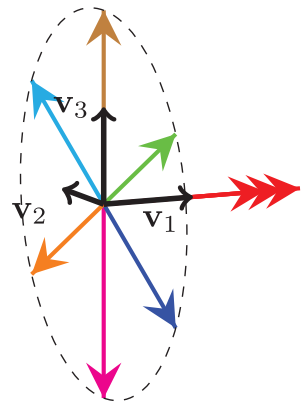

(b)

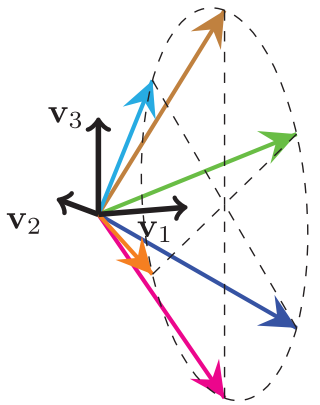

(c)

Figure 4. Visualization of minimal sets of discrete directions representing extremal transversely isotropic orientation states which are $\mathbf{N}$-isotropic: (a), (b) two views on $\mathcal{D}_{\mathbb{A}}$ leading to $\mathbb{A}$, (c) $\mathcal{D}_{\mathbb{B}}$ leading to $\mathbb{B}$.

holds, but the states differ significantly. Figure 3 shows that for the special case of transversely isotropy, only two second-order fiber orientation tensors, namely those defined by $\alpha=-1 / 3$ and $\alpha=2 / 3$, are connected to a corresponding fourth-order fiber orientation tensor by a one-to-one relation. The subsequent sections show that for weaker symmetries a one-to-one correspondence solely remains for the unidirectional case. Closure approximations, briefly discussed in Section 2.6, construct a one-to-one mapping between a given second-order fiber orientation tensor and an unknown fourth-order fiber orientation tensor. In Figure 3, three closures which are discussed in Section 2.6 are visualized and indicate that the variety of transversely isotropic fourth-order fiber orientation tensors is not fully reflected by the selected closures.

An intuitive interpretation of the orientation states described by $\mathbb{A}$ and $\mathbb{B}$ in Equations (69) and (70) is obtained by minimal sets of discrete fibers which are described by these fiber orientation tensors. The single fibers can be visualized and represent minimal realisations of states in $\mathcal{N}^{\text {transv }}$. To the best of the authors' knowledge, visualization of fiber orientation tensors by minimal discrete fiber arrangements is new. For both the state $\mathbb{A}$ and the state $\mathbb{B}$, an ODF can be build by a sum of delta functions with homogeneous weights

$$
\psi(\mathbf{n})=\frac{1}{N} \sum_{i}^{N} \frac{1}{2}\left(\delta\left(\mathbf{n}-\mathbf{n}_{i}\right)+\delta\left(\mathbf{n}+\mathbf{n}_{i}\right)\right)
$$

based on a set of discrete fiber directions $\left\{\mathbf{n}_{i}\right.$ with $\left.i \in[1, \ldots, N]\right\}$. Minimal sets of discrete fiber directions leading to orientation tensors $\mathbb{A}$ and $\mathbb{B}$ are

$$
\begin{aligned}
& \mathcal{D}_{\mathbb{A}}=\left\{\left[\begin{array}{c}
0 \\
\cos \left(\phi_{i}\right) \\
\sin \left(\phi_{i}\right)
\end{array}\right] \text { with } \phi_{i}=\frac{2 \pi(i-1)}{6} \text { for } i \in[1, \ldots, 6]\right\}+3\left\{\left[\begin{array}{l}
1 \\
0 \\
0
\end{array}\right]\right\} \text { and } \\
& \mathcal{D}_{\mathbb{B}}=\left\{\left[\begin{array}{c}
\cos (\pi / 4) \\
\cos \left(\phi_{i}\right) \\
\sin \left(\phi_{i}\right)
\end{array}\right] \text { with } \phi_{i}=\frac{2 \pi(i-1)}{6} \text { for } i \in[1, \ldots, 6]\right\}
\end{aligned}
$$

given in the orientation coordinate system $\left\{\mathbf{v}_{i}\right\}$. Visualizations of the sets of directions are given in Figures $4(\mathrm{~b})$ and (c). Comparison of the vector sets and inspection of Figures 4(b) and (c) and 3 allow for an interpretation of the parameter $\rho$ in Equation (64). The lower the value of $\rho$, the more pronounced are directions towards diagonals between the principle axis of the orientation coordinate system.

2.5.4. Orthotropic case. An orthotropic harmonic tensor of fourth-order is given by

$$
\mathbb{F}^{\text {ortho }}\left(d_{1}, d_{2}, d_{3}\right)=\mathbb{F}^{\text {tricl }}\left(d_{1}, d_{2}, d_{3}, 0,0,0,0,0,0\right)
$$

with $\mathbb{F}^{\text {tricl }}$ defined in Equation (60). As the weakest material symmetry of second-order tensors is orthotropy, a parameterization of orthotropic fourth-order orientation tensors is given by

$$
\mathbb{N}^{\text {ortho }}\left(\mathbf{N}, d_{1}, d_{2}, d_{3}\right)=\mathbb{N}^{\text {iso }}+\frac{6}{7} \operatorname{sym}\left(\operatorname{dev}(\mathbf{N}) \otimes \mathbf{I}_{2}\right)+\mathbb{F}^{\text {ortho }}\left(d_{1}, d_{2}, d_{3}\right) .
$$


The set of admissible orthotropic fourth-order orientation tensors is

$$
\mathcal{N}^{\text {ortho }}=\left\{\mathbb{N}^{\text {ortho }}\left(\mathbf{N}, d_{1}, d_{2}, d_{3}\right) \mid 0 \leq \Lambda_{i} \forall \Lambda_{i} \text { in eigenvalues of } \mathbb{N}^{\text {ortho }}\right\},
$$

see Section 2.5.2. Parameterization of $\mathbf{N}$ following Equation (28) leads to

$$
\begin{aligned}
\mathbb{N}^{\text {ortho }}\left(\alpha_{1}, \alpha_{3}, d_{1}, d_{2}, d_{3}\right) & =\mathbb{N}^{\text {iso }} \\
& +\left[\begin{array}{lll|lll}
\frac{3}{7}\left(2 \alpha_{1}-\alpha_{3}\right)-d_{1}-d_{2} & \frac{1}{14}\left(\alpha_{1}-2 \alpha_{3}\right)+d_{1} & \frac{1}{14}\left(\alpha_{1}+\alpha_{3}\right)+d_{2} & 0 & 0 & 0 \\
& -\frac{3}{7}\left(\alpha_{1}+\alpha_{3}\right)-d_{1}-d_{3} & \frac{1}{14}\left(-2 \alpha_{1}+\alpha_{3}\right)+d_{3} & 0 & 0 & 0 \\
& & \frac{34}{7}\left(-\alpha_{1}+2 \alpha_{3}\right)-d_{2}-d_{3} & 0 & 0 & 0 \\
\hline & \text { completely } & \text { symmetric }
\end{array}\right] \mathbf{B}_{\xi}^{\mathbf{v}} \otimes \mathbf{B}_{\zeta}^{\mathbf{v}} .
\end{aligned}
$$

Three eigenvalues of $\mathbb{N}^{\text {ortho }}$ in the six-dimensional space of the Mandel representation are directly given by

$$
\begin{aligned}
& \frac{1}{2} \Lambda_{1}=\frac{1}{14}\left(-2 \alpha_{1}+\alpha_{3}\right)+d_{3}+\frac{1}{15} \\
& \frac{1}{2} \Lambda_{2}=\frac{1}{14}\left(\alpha_{1}+\alpha_{3}\right)+d_{2}+\frac{1}{15} \\
& \frac{1}{2} \Lambda_{3}=\frac{1}{14}\left(\alpha_{1}-2 \alpha_{3}\right)+d_{1}+\frac{1}{15}
\end{aligned}
$$

due to the diagonal form of the lower right quadrant of $\mathbb{N}^{\text {ortho }}$ in Equation (78). Following [35, equation (5.35)], the remaining eigenvalues $\left(\Lambda_{4}, \Lambda_{5}, \Lambda_{6}\right)$ are the roots of the characteristic polynomial given by

$\operatorname{det}\left[\begin{array}{ccc}\frac{3}{7}\left(2 \alpha_{1}-\alpha_{3}\right)-d_{1}-d_{2}+\frac{1}{5}-\Omega & \frac{1}{14}\left(\alpha_{1}-2 \alpha_{3}\right)+d_{1}+\frac{1}{15} & \frac{1}{14}\left(\alpha_{1}+\alpha_{3}\right)+d_{2}+\frac{1}{15} \\ & -\frac{3}{7}\left(\alpha_{1}+\alpha_{3}\right)-d_{1}-d_{3}+\frac{1}{5}-\Omega & \frac{1}{14}\left(-2 \alpha_{1}+\alpha_{3}\right)+d_{3}+\frac{1}{15} \\ & \frac{3}{7}\left(-\alpha_{1}+2 \alpha_{3}\right)-d_{2}-d_{3}+\frac{1}{5}-\Omega\end{array}\right]=0$

being cubic in $\Omega$. For transversely isotropic orientation tensors, a compact and explicit representation of the admissible parameters, is given by Equation (65). For the orthotropic case, explicit formulas specifying $\mathcal{N}^{\text {ortho }}$ by combinations of the parameters of $\mathbb{N}^{\text {ortho }}$, can be identified analytically, e.g., using complex or trigonometric expressions. As an alternative, non-negativity of eigenvalues can be demanded by alternating signs of monomials of the cubic characteristic following Vieta's formula preventing the need to explicitly calculate the eigenvalues. However, the resulting expressions are lengthy and, therefore, are not given here. For applications $\mathcal{N}^{\text {ortho }}$ can be calculated numerically.

However, for a given specific second-order orientation tensor $\mathbf{N}$, compact representations of sets of admissible tuples $\left(d_{1}, d_{2}, d_{3}\right)$, i.e., tuples leading to $\mathbb{N}^{\text {ortho }}\left(\mathbf{N}, d_{1}, d_{2}, d_{3}\right) \in \mathcal{N}^{\text {ortho }}$, exist. For reference, an explicit parameterization of admissible tuples for the special case of an isotropic second-order orientation tensor $\mathbf{N}^{\text {iso }}$ is given in Appendix D. Admissible tuples for specific $\mathbf{N}$ along three paths are visualized in Figures 6, 7 and 8. The paths are defined in Figure 5(a).

Figure 6 reveals that the admissible region of $\left(d_{1}, d_{2}, d_{3}\right)$ for an isotropic second-order orientation tensor is symmetric and comparably large. Departing from the isotropic second-order orientation tensor along the blue path along $\mathbf{v}_{1}$ (see Figure 5(a)) towards the unidirectional state, the admissible region of $\left(d_{1}, d_{2}, d_{3}\right)$ changes its shape and position in the space spanned by $d_{1}, d_{2}$ and $d_{3}$ and finally degenerates to a single point representing the unidirectional state. As all points on the blue path are transversely isotropic with respect to the $\mathbf{v}_{1}$-axis, the admissible ranges of the parameters $d_{1}$ and $d_{2}$ are identical along this path (see $\mathbb{F}^{\text {transv1 }}$ in Equation (27)). Figure 7 contains views on the admissible tuples for specific second-order orientation tensors along the orange path, in 5(a). Second-order orientation tensors along the orange path between the isotropic and the planar isotropic state are transversely isotropic with principle axis $\mathbf{v}_{3}$. Therefore, admissible ranges of the parameters $d_{2}$ and $d_{3}$ change homogeneously and shrink along the path towards the planar isotropic second-order orientation tensor. For the planar isotropic second-order orientation tensor, the parameters $d_{2}$ and $d_{3}$ are fixed and equal to $-3 / 70$. Therefore, at this second-order state the admissible region degenerates to a line and the remaining degree of freedom of an orthotropic fourth-order orientation tensor with planar isotropic $\mathbf{N}^{\text {planar iso }}$ is $d_{1}$ with admissible 

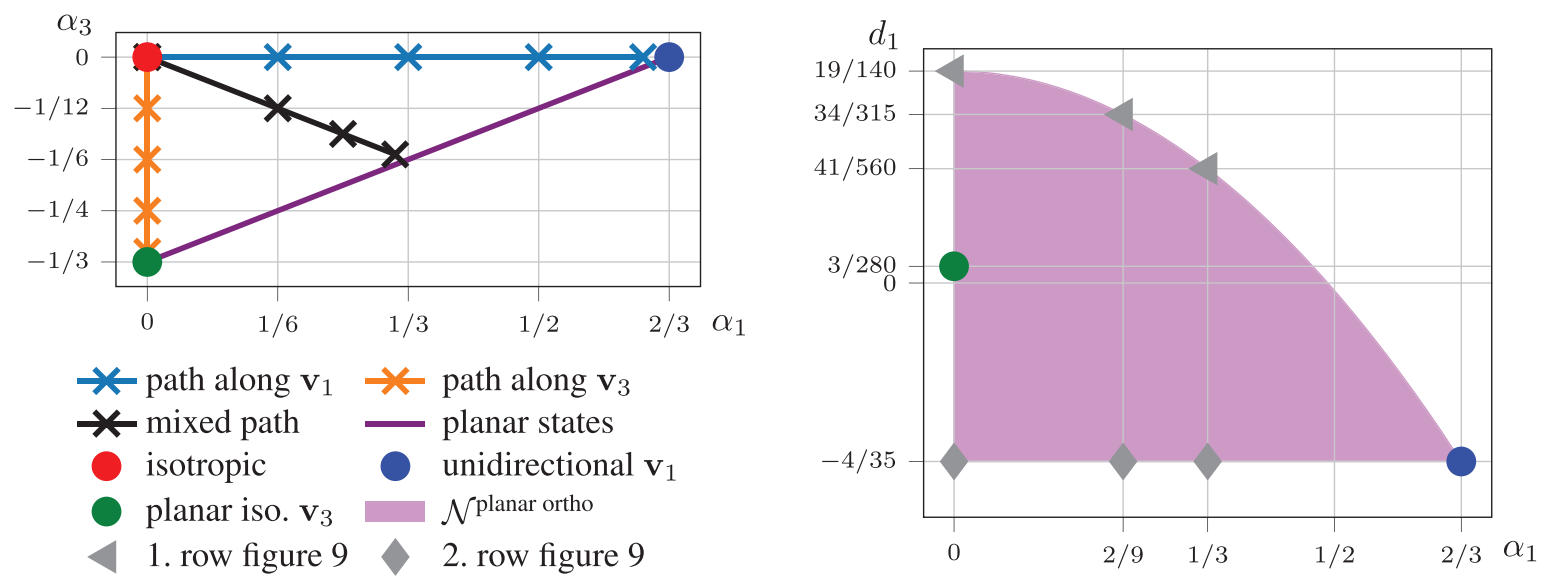

$*$ path along $\mathbf{v}_{1} \quad *$ path along $\mathbf{v}_{3}$

$*$ mixed path

isotropic

- planar states

planar iso. $\mathbf{v}_{3}$

unidirectional $\mathbf{v}_{1}$

1. row figure 9 $\mathcal{N}$ planar ortho

2. row figure 9

(b)

(a)

Figure 5. (a) Paths on the orientation triangle defining specific second-order orientation tensors used in Figures 6, 7, and 8. (b) Admissible ranges of $d_{1}$ for planar orthotropic orientation states and specific orientation states used in Figure 9 . The legend is shared with Figure 5(a).
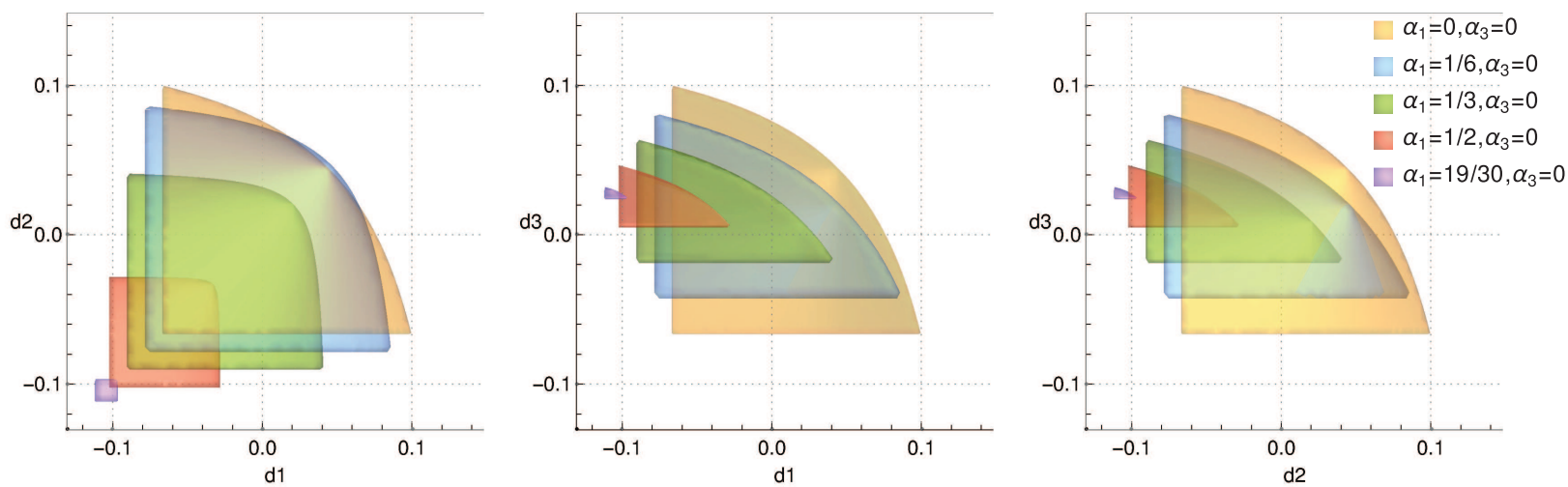

Figure 6. Three views on admissible ranges of $\left(d_{1}, d_{2}, d_{3}\right)$ for specific second-order orientation tensors specified along the blue path (along $\mathbf{v}_{1}$ ) in Figure 5(a).
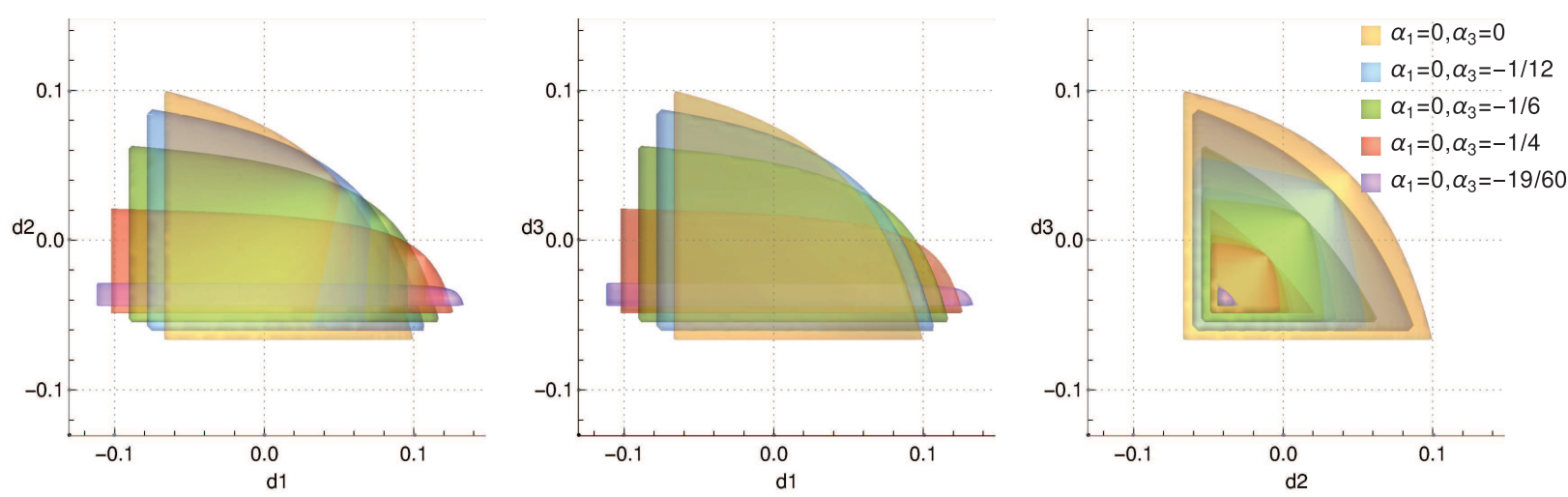

Figure 7. Three views on admissible ranges of $\left(d_{1}, d_{2}, d_{3}\right)$ for specific second-order orientation tensors specified along the orange path (along $\mathbf{v}_{3}$ ) in Figure 5(a).

range $-4 / 35 \leq d_{1} \leq 19 / 140$. The planar isotropic orientation state is located at $d_{1}=3 / 280$. Second-order orientation tensors of planar orientation states are located at the purple line in Figure 5(a). This line connects the planar isotropic and the unidirectional states. Planar orthotropic fourth-order orientation tensors are degenerated 

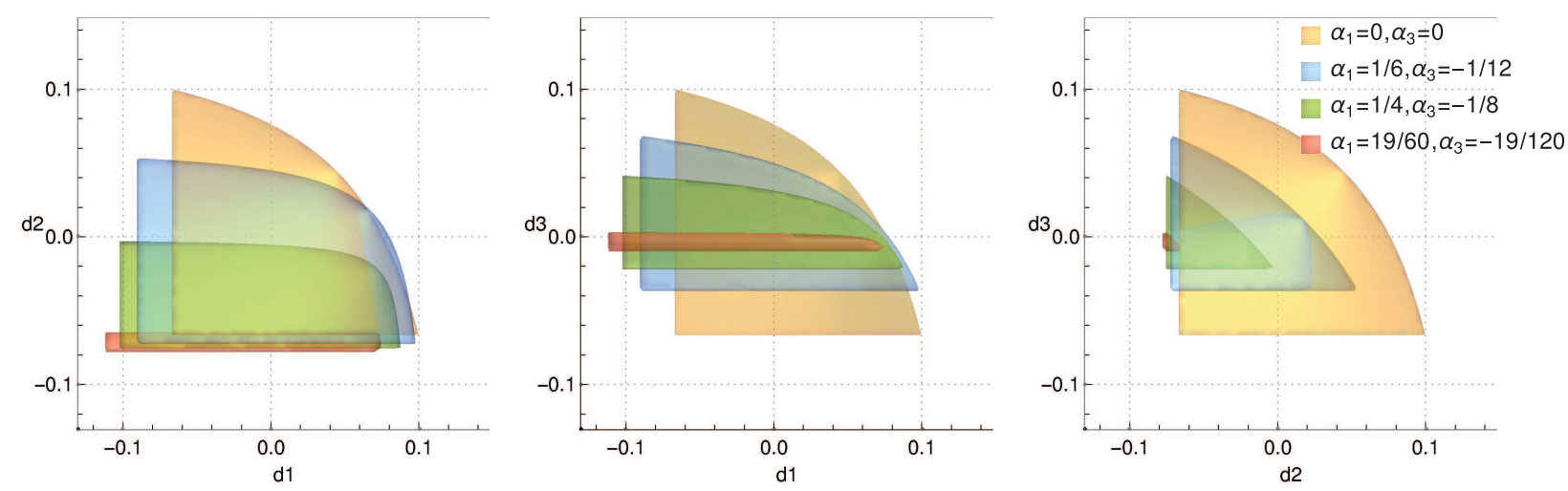

Figure 8. Three views on admissible ranges of $\left(d_{1}, d_{2}, d_{3}\right)$ for specific second-order orientation tensors specified along the black path on orthotropic states in Figure 5(a).

in the sense that the parameters $d_{2}$ and $d_{3}$ are directly related to $\mathbf{N}$, ensuring that the orientation state is planar. As a consequence, the remaining degree of freedom of planar orthotropic fourth-order orientation tensors is solely determined by the remaining parameter $d_{1}$. In consequence, a parameterization of planar orthotropic fourth-order orientation tensors is given by

$$
\mathbb{N}^{\text {planar ortho }}\left(\alpha_{1}, d_{1}\right)=\mathbb{N}^{\text {ortho }}\left(\mathbf{N}=\mathbf{N}^{\text {planar }}\left(\alpha_{1}\right), d_{1}, d_{2}=\frac{-15 \alpha_{1}-6}{140}, d_{3}=\frac{15 \alpha_{1}-6}{140}\right)
$$

with $\mathbf{N}^{\text {planar }}\left(\alpha_{1}\right)$ following Equation (35). The set of admissible planar orthotropic orientation tensors

$$
\mathcal{N}^{\text {planar ortho }}=\left\{\mathbb{N}^{\text {planar ortho }}\left(\alpha_{1}, d_{1}\right) \mid 0 \leq \alpha_{1} \leq \frac{2}{3},-\frac{4}{35} \leq d_{1} \leq \frac{19}{140}-\frac{9}{16} \alpha_{1}^{2}\right\}
$$

is visualized in Figure 5(b). For reference, the planar isotropic and unidirectional states are highlighted in Figure 5(b). To the best of the authors' knowledge, the set of admissible orthotropic fiber orientation tensors of fourth order has not been presented in the literature before. Second-order orientation tensors which describe planar orientation states in a three-dimensional space have four variable parameters. Three of these four parameters define the mapping $\mathbf{Q}$ (see Equation (22)) which defines the orientation coordinate system and the fourth parameter defines the orientation state. One possible choice for this fourth parameter is $\alpha_{1}$ (see Equation (35)). Orthotropic fourth-order orientation tensors, which represent planar orientation states, add one additional independent parameter, e.g., $d_{1}$. Figure 5(b) visualizes the admissible ranges of $d_{1}$ as a function of $\alpha_{1}$, assuming orthotropy. The admissible range of this fourth-order orientation tensor parameter $d_{1}$ degenerates to a single value in case of the unidirectional state. This degeneration reveals a valuable view on the information which is contained in $\mathbb{N}$ but not in $\mathbf{N}$. The second-order orientation tensor $\mathbf{N}$ contains directional measures in the directions defined by the axes of the orientation coordinate system. An orthotropic fourth-order orientation tensor contains additional directional information representing the spread around the directions defined by the axes of the orientation coordinate system. For the unidirectional state, i.e., if all fibers are aligned in one direction, there is no spread. In the planar orthotropic case, there is only one spread. This mental model is a simplification of directional measures by spherical harmonics but suits engineering applications and can be used to interpret Figures 6-8. In the following, examples of planar orthotropic orientation states are discussed. Figures 9(a) $-9(\mathrm{~g})$ visualize sets of discrete directions for planar orthotropic orientation states marked in Figure 5(b). The upper (lower) row of subfigures in Figure 9 represents discrete sets of directions which lead to planar and orthotropic fourth-order orientation tensors with maximum (minimum) admissible values $d_{1}$ (see Figure 5(b) for reference). A minimal set of discrete directions for each subfigure in Figure 9 is given in the caption. The discrete sets of directions labeled $\mathcal{D}^{(i)}$ for $i \in[\mathrm{a}, \mathrm{b}, \mathrm{c}, \mathrm{d}, \mathrm{e}, \mathrm{f}, \mathrm{g}]$ in the caption of Figure 9 can be combined with Equation (72) to identify the corresponding ODF and orientation tensors. For reference, limiting cases of orthotropic fourth-order orientation tensors which contract to the planar isotropic second-order orientation tensor are given 


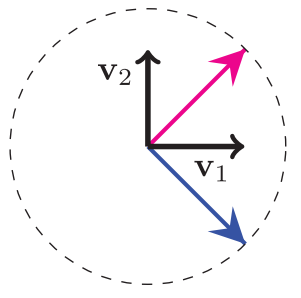

(a)

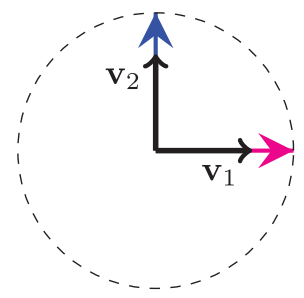

(b)

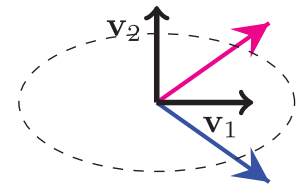

(c)

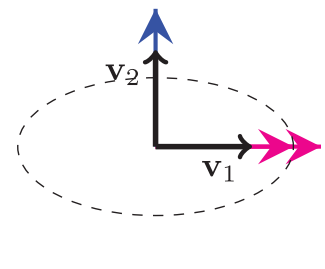

(d)

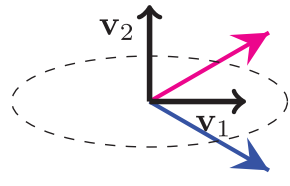

(e)

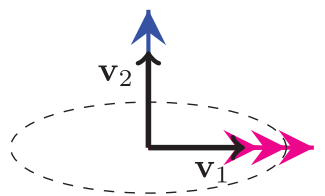

(f)

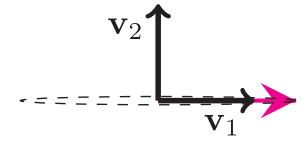

(g)

Figure 9. Visualizations of minimal discrete sets of directions in the orientation coordinate system $\left\{\mathbf{v}_{i}\right\}$ for given planar orthotropic orientation tensors which are marked in Figure 9 and parameterized by $\alpha_{1}$ and $d_{1}$ (see Equation (83)): (a) $\alpha_{1}=0, \quad d_{1}=19 / 140$ with $\mathcal{D}^{(\mathrm{a})}=\{[1 / \sqrt{2}, 1 / \sqrt{2}-\varepsilon, 0],[1 / \sqrt{2},-(1 / \sqrt{2}-\varepsilon), 0]\}$ with $\varepsilon \ll 1 ;$ (b) $\alpha_{1}=0, d_{1}=-4 / 35$ with $\mathcal{D}^{(b)}=\{[1,0,0],[0,1,0]\} ;$ (c) $\alpha_{1}=2 / 9, d_{1}=34 / 315$ with $\mathcal{D}^{(\mathrm{c})}=\{[\sqrt{2} / \sqrt{3}, 1 / \sqrt{3}, 0],[\sqrt{2} / \sqrt{3},-1 / \sqrt{3}, 0]\}$; (d) $\alpha_{1}=2 / 9$, $d_{1}=-4 / 35$ with $\mathcal{D}^{(\mathrm{d})}=\{2 \times[1,0,0],[0,1,0]\} ;(\mathrm{e}) \alpha_{1}=1 / 3, d_{1}=41 / 560$ with $\mathcal{D}^{(\mathrm{e})}=\{[\sqrt{3} / 2,1 / 2,0],[\sqrt{3} / 2,-1 / 2,0]\} ;$ (f) $\alpha_{1}=1 / 3, d_{1}=-4 / 35$ with $\mathcal{D}^{(\mathrm{f})}=\{3 \times[1,0,0],[0,1,0]\} ;(\mathrm{g}) \alpha_{1}=2 / 3, d_{1}=-4 / 35$ with $\mathcal{D}^{(\mathrm{g})}=\{[1,0,0]\}$.

by

$$
\mathbb{N}^{\text {planar ortho }}\left(\alpha_{1}=0, d_{1}=19 / 140\right)=\left[\begin{array}{rrr|rrr}
1 / 4 & 1 / 4 & 0 & 0 & 0 & 0 \\
1 / 4 & 0 & 0 & 0 & 0 \\
& 0 & 0 & 0 & 0 \\
\hline \text { completely } & \text { symmetric }
\end{array}\right] \mathbf{B}_{\xi}^{\mathbf{v}} \otimes \mathbf{B}_{\zeta}^{\mathbf{v}}
$$

and

$$
\mathbb{N}^{\text {planar ortho }}\left(\alpha_{1}=0, d_{1}=-4 / 35\right)=\left[\begin{array}{ccc|ccc}
1 / 2 & 0 & 0 & 0 & 0 & 0 \\
1 / 2 & 0 & 0 & 0 & 0 \\
& 0 & 0 & 0 & 0 \\
\hline \text { completely } & \text { symmetric }
\end{array}\right] \mathbf{B}_{\xi}^{\mathbf{v}} \otimes \mathbf{B}_{\zeta}^{\mathbf{v}} .
$$

2.5.5. Planar case. Without loss of generality, inspection of planar states is restricted to orientation states which are located inside the plane spanned by $\mathbf{v}_{1}$ and $\mathbf{v}_{2}$. Following this convention, some coefficients of moment tensors represented in the orientation coordinate system (see, e.g., Equation (53) or (102)) vanish, because outof-plane components of the unit vector, which builds the moment tensors, equal zero. Combining the knowledge of vanishing coefficients of $\mathbb{N}^{\text {planar }}$ with Equation (59) leads to the parameterization

$$
\mathbb{N}^{\text {planar }}\left(\alpha_{1}, d_{1}, d_{8}\right)=\left[\begin{array}{ccc|ccc}
\frac{3}{4} \alpha_{1}-d_{1}+\frac{27}{70} & d_{1}+\frac{4}{35} & 0 & 0 & 0 & \sqrt{2} d_{8} \\
& -\frac{3}{4} \alpha_{1}-d_{1}+\frac{27}{70} & 0 & 0 & 0 & -\sqrt{2} d_{8} \\
\text { completely } & 0 & 0 & 0 & 0 \\
\hline & & \text { symmetric }
\end{array}\right] \mathbf{B}_{\xi}^{\mathbf{v}} \otimes \mathbf{B}_{\zeta}^{\mathbf{v}},
$$




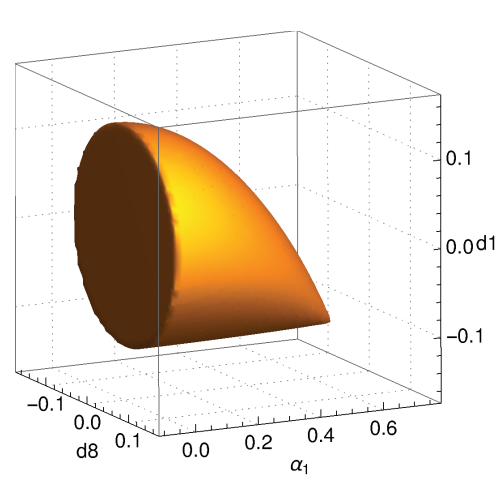

(a)

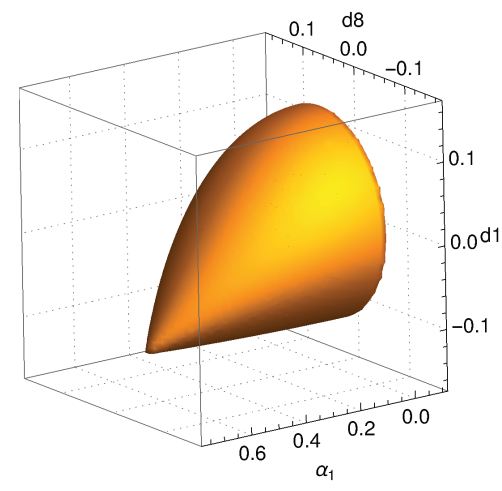

(b)

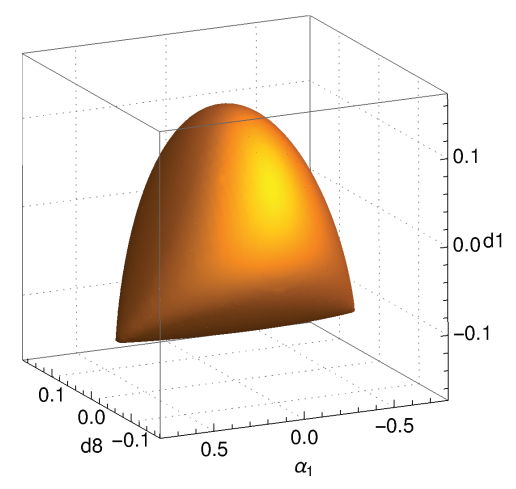

(c)

Figure 10. (a), (b) Views on $\mathcal{N}^{\text {planar }}$. (c) Transition of admissible parameters of planar states between two unidirectional orientation states visualized by varying the parameter $\alpha_{1}$ in Equation (89) in the range $-2 / 3 \leq \alpha_{1} \leq 2 / 3$.

which fits into the general triclinic framework given by Equations (59) and (60) with

$$
\mathbb{N}^{\text {planar }}\left(\alpha_{1}, d_{1}, d_{8}\right)=\mathbb{N}\left(\mathbf{N}^{\text {planar }}\left(\alpha_{1}\right), d_{1}, d_{2}=\frac{-15 \alpha_{1}-6}{140}, d_{3}=\frac{15 \alpha_{1}-6}{140}, 0,0,0,0, d_{8}, d_{9}=-d_{8}\right) .
$$

Similar to the planar orthotropic case, the parameters $d_{2}$ and $d_{3}$ are directly related to $\mathbf{N}$, ensuring that out-ofplane coefficients in the upper-left quadrant in Equation (87) vanish. Removing the orthotropic constraint adds degrees of freedom. However, the planar plane intrinsically acts as a symmetry plane and planar orientation states have at least monoclinic material symmetry. As a consequence of this material symmetry as well as due to the planarity itself, the parameters $d_{4}, d_{5}, d_{6}$ and $d_{7}$ in Equation (60), which is utilized in Equation (88), equal zero. Owing to the planarity, $d_{9}=-d_{8}$ holds in Equation (88) as the coefficient $\sqrt{2}\left(d_{8}+d_{9}\right)$ in Equation (60) has to vanish. This can be seen by comparison of Equation (60) and the moment tensor in Equation (102) where the planarity implies $\theta=\frac{\pi}{2}$. Demanding positive eigenvalues of $\mathbb{N}^{\text {planar }}$ in equation (87) leads to the set of admissible planar fourth-order orientation tensors

$$
\mathcal{N}^{\text {planar }}=\left\{\mathbb{N}^{\text {planar }}\left(\alpha_{1}, d_{1}, d_{8}\right) \mid 0 \leq \alpha_{1} \leq \frac{2}{3},-\frac{4}{35} \leq d_{1} \leq \frac{19}{140}-\frac{9}{16} \alpha_{1}^{2},-f\left(\alpha_{1}, d_{1}\right) \leq d_{8} \leq f\left(\alpha_{1}, d_{1}\right)\right\}
$$

with

$$
f\left(\alpha_{1}, d_{1}\right)=\frac{1}{140} \sqrt{304-1260 \alpha_{1}^{2}+420 d_{1}-11025 \alpha_{1}^{2} d_{1}-19600 d_{1}^{2}} .
$$

To the best of the author's knowledge, this set is new in literature and of great practical importance for shelllike components reinforced with long fibers. The set of admissible planar fourth-order orientation tensors is visualized in Figures 10(a) and 10(b). The connection to the admissible ranges in the planar orthotropic case, which is illustrated in Figure 5(b), is given by a projection of $\mathcal{N}^{\text {planar }}$ onto the plane $d_{8}=0$. In Figure $10(\mathrm{c})$, the plotting range of the parameter $\alpha_{1}$ is extended from one unidirectional state along a planar boundary of the orientation plane in Figure 2(b) towards a second unidirectional orientation state. This illustrates the continuity of the admissible ranges and the redundancy of the orientation plane.

\subsection{A note on closure approximations}

For a given second-order orientation tensor $\mathbf{N}$, closure approximations identify an unknown fourth-order orientation tensor $\mathbb{N}^{\text {unknown }}$ following a specific closure approach such that

$$
\mathbb{N}^{\text {unknown }}\left[\mathbf{I}_{2}\right]=\mathbf{N}
$$


holds. Based on the quantity which is directly affected by a closure's assumptions, closure approximations might be classified into three groups. The first group contains closures based on algebraic assumptions. Closures based on assumptions on the material symmetry of orientation tensors form the second group. The third group contains closures which state assumptions on the ODF. The linear closure is given by

$$
\mathbb{N}^{\text {linear }}(\mathbf{N})=\mathbb{N}^{\text {iso }}+\frac{6}{7} \operatorname{sym}\left(\operatorname{dev}(\mathbf{N}) \otimes \mathbf{I}_{2}\right),
$$

belongs to the first group and is defined in [17, Equation (13)] or [16]. The representation in (92) benefits from the notation introduced in Equations (56) and (57). The linear closure states that the unknown fourthorder orientation tensor is linear in the second-order orientation tensor. This implies vanishing fourth-order harmonic part, i.e., dev $\left(\mathbb{N}^{\text {unknown }}\right)=\mathbb{O}$. Figure 3 contains the linear closure as dashed light green line and clearly shows the limitations of this closure as the line is not completely within the admissible parameter space. The second group contains, e.g., orthotropic fitted closures (ORFs) which combine analytical insights on the material symmetry of fiber orientation tensors with fitting procedures reproducing selected flow simulation results. Examples are given in $[8,9,48]$ and both the ORF [8] and the invariant-based optimal fitting closure (IBOF) [9] are visualized in Figure 3 using [49]. The third group contains closures, e.g., [4,18], which enable exact reconstructions of the unknown ODF, based on the assumption that the ODF belongs to a special class of ODFs. Therefore, assumptions are made on the ODF and tensors of any order can be constructed based on the reconstructed ODF. Although this short note on closures does not claim to be exhaustive, reference is made to additional closures, e.g., [50-52]. Reproduction of the ODF by maximization of the information entropy, e.g., [5] (see also [22]) states an alternative and its wording explicitly addresses the problem of gaining information by assumptions. Assessment of closure approximations is challenging because the definition of an application-independent metric for the quality of the closure is difficult. Nevertheless, assessments of closure approximations are given in $[14,15,19]$.

\section{Summary and conclusions}

Application of linear invariant decomposition with focus on index symmetry [42] transfers the results of Kanatani [1] into the continuum mechanics framework and leads to a compact representation of fourth-order fiber orientation tensors in Equations (56) and (57). A harmonic, i.e., completely symmetric and completely traceless, triclinic fourth-order structure tensor is introduced in Equation (60). This structure tensor has a simplified structure in the coordinate system spanned by the eigenvectors of the second-order fiber orientation tensor and leads to a parameterization of generic fourth-order fiber orientation tensors in Equation (59). Material symmetries of the ODF reduce the number of independent parameters. In the triclinic case, the independent parameters are a second-order fiber orientation tensor plus nine scalars. Admissible ranges of the independent parameters are discussed in detail for orthotropy, transversely isotropy, and planar cases. Insufficiency of coefficient-wise constraints are demonstrated for the transversely isotropic case. The variety of fourth-order fiber orientation tensors is given by the set of positive-definite tensors which can be expressed by Equation (59). Inspection of planar orthotropic states illustrates the character of fourth-order orientation information. Notes on closure approximations demonstrate their limitations on reflecting the variety of fourth-order fiber orientation tensors. As a summary, the parameterizations of generic second-order orientation tensors $\mathbf{N}$

$$
\mathbf{N}\left(\alpha_{1}, \alpha_{3}\right)=\mathbf{N}^{\text {iso }}+\alpha_{1} \mathbf{F}^{\text {transv1 }}+\alpha_{3} \mathbf{F}^{\text {transv3 }}
$$

generic fourth-order orientation tensors $\mathbb{N}$

$$
\mathbb{N}\left(\mathbf{N}, d_{1}, \ldots, d_{9}\right)=\mathbb{N}^{\text {iso }}+\frac{6}{7} \operatorname{sym}\left(\operatorname{dev}(\mathbf{N}) \otimes \mathbf{I}_{2}\right)+\mathbb{F}^{\text {tricl }}\left(d_{1}, \ldots, d_{9}\right),
$$

orthotropic fourth-order orientation tensors $\mathbb{N}^{\text {ortho }}$

$$
\mathbb{N}^{\text {ortho }}\left(\mathbf{N}, d_{1}, d_{2}, d_{3}\right)=\mathbb{N}^{\text {iso }}+\frac{6}{7} \operatorname{sym}\left(\operatorname{dev}(\mathbf{N}) \otimes \mathbf{I}_{2}\right)+\mathbb{F}^{\text {ortho }}\left(d_{1}, d_{2}, d_{3}\right)
$$


and planar fourth-order orientation tensors $\mathbb{N}^{\text {planar }}$

$$
\mathbb{N}^{\text {planar }}\left(\alpha_{1}, d_{1}, d_{8}\right)=\mathbb{N}\left(\mathbf{N}^{\text {planar }}\left(\alpha_{1}\right), d_{1}, d_{2}=\frac{-15 \alpha_{1}-6}{140}, d_{3}=\frac{15 \alpha_{1}-6}{140}, 0,0,0,0, d_{8}, d_{9}=-d_{8}\right)
$$

are repeated, including the structure tensors

$$
\begin{aligned}
& \mathbf{F}^{\text {transv1 }}=\left[\begin{array}{ccc}
1 & 0 & 0 \\
& -1 / 2 & 0 \\
\text { sym } & & -1 / 2
\end{array}\right] \mathbf{v}_{i} \otimes \mathbf{v}_{j} \quad \text { and } \quad \mathbf{F}^{\text {transv3 }}=\left[\begin{array}{ccc}
-1 / 2 & 0 & 0 \\
& -1 / 2 & 0 \\
\text { sym } & & 1
\end{array}\right] \mathbf{v}_{i} \otimes \mathbf{v}_{j}, \\
& \mathbb{F}^{\text {tricl }}\left(d_{1}, \ldots, d_{9}\right)=\left[\begin{array}{lll|lll}
-\left(d_{1}+d_{2}\right) & d_{1} & d_{2} & -\sqrt{2}\left(d_{4}+d_{5}\right) & \sqrt{2} d_{6} & \sqrt{2} d_{8} \\
& -\left(d_{1}+d_{3}\right) & d_{3} & \sqrt{2} d_{4} & -\sqrt{2}\left(d_{6}+d_{7}\right) & \sqrt{2} d_{9} \\
& & -\left(d_{2}+d_{3}\right) & \sqrt{2} d_{5} & \sqrt{2} d_{7} & -\sqrt{2}\left(d_{8}+d_{9}\right) \\
\hline & & & \multicolumn{3}{|c}{\text { symmetric }}
\end{array}\right] \mathbf{B}_{\xi}^{\mathbf{v}} \otimes \mathbf{B}_{\zeta}^{\mathbf{v}},
\end{aligned}
$$

and

$$
\mathbb{F}^{\text {ortho }}\left(d_{1}, d_{2}, d_{3}\right)=\mathbb{F}^{\text {tricl }}\left(d_{1}, d_{2}, d_{3}, 0,0,0,0,0,0\right) \text {. }
$$

Admissible parameter ranges follow from the requirement of positive semi-definiteness (see Equation (62)) and are explicitly given for special cases of material symmetry in Equations (65), (84), (89), and (106).

In addition to the review contribution, novel technical and scientific contributions in this work are listed hereafter.

- An invariant framework for parameterizations of fourth-order fiber orientation tensors based on deviators from the isotropic orientation state is developed. The most general case is obtained by an orthotropic deviator of second order and an triclinic deviator of fourth order. Within this framework, deviators of stronger material symmetries are obtained as special cases with constrained parameters.

- $\quad$ Explicit parameterizations and admissible parameter ranges present in the literature are extended by orthotropic as well as planar fiber orientation tensors in a three-dimensional framework.

- Visualizations of admissible parameter sets are given for the first time for the orthotropic states in Figures 5-8 and for the planar states in Figures 10(a).

- Positive semi-definiteness is identified to be a stronger constraint on admissible orientation tensors than linear constraints on tensor coefficients which themselves are identified to be stronger constraints than a bonded norm.

- A short hand notation for completely symmetric fourth-order tensors is introduced.

- Representations of discrete fiber sets (Figures 4 and 9) are identified to be an valuable visualization of orientation tensors.

These results enable engineers to parameterize the space of admissible fiber orientation tensors obtaining a valuable tool for engineering applications such as model inspection or data validation.

\section{Author Contributions}

Julian Karl Bauer: Conceptualization, methodology software, validation, formal analysis, investigation, resources, writing-original draft preparation, writing-review and editing, visualization, Thomas Böhlke: Conceptualization, methodology formal analysis, writing-review and editing, supervision, project administration, funding acquisition. All authors have read and agreed to the published version of the manuscript.

\section{Acknowledgments}

We thank the anonymous reviewer whose critical review helped to improve and clarify this manuscript. Provision of the open source Python package [49] by Nils Meyer is gratefully acknowledged. Review by Jonas Hund is gratefully acknowledged. 


\section{Declaration of conflicting interests}

The authors declare no conflict of interest. The funders had no role in the design of the study; in the collection, analyses, or interpretation of data; in the writing of the manuscript, or in the decision to publish the results.

\section{Funding}

The research documented in this manuscript has been funded by the German Research Foundation (DFG) within the International Research Training Group "Integrated engineering of continuous-discontinuous long fiber-reinforced polymer structures" (GRK 2078/2). The support by the German Research Foundation (DFG) is gratefully acknowledged.

\section{Code}

Selected parts of the code used to create this work are openly available at https://github.com/JulianKarlBauer/fiber_orientation_tensors_ 2021. Closure approximations in this work are based on [49].

$\begin{array}{ll}\text { Abbreviations } & \\ \text { ODF } & \text { Fiber orientation distribution function } \\ \text { CODF } & \text { Crystal orientation distribution function } \\ \text { IBOF } & \text { Invariant-based optimal fitting } \\ \text { transv. } & \text { Transversely isotropic } \\ \text { RV } & \text { Reference volume } \\ \text { ORF } & \text { Orthotropic fitted } \\ \text { unidirect. } & \text { Unidirectional } \\ \text { plan. } & \text { Planar } \\ \text { sym } & \text { Symmetric } \\ \text { perm } & \text { Permutation } \\ \text { iso } & \text { Isotropic } \\ \text { ortho } & \text { Orthotropic }\end{array}$

\section{ORCID iD}

Julian Karl Bauer (iD https://orcid.org/0000-0002-4931-5869

Thomas Böhlke (iD https://orcid.org/0000-0001-6884-0530

\section{References}

[1] Kanatani, KI. Distribution of directional data and fabric tensors. Int J Eng Sci 1984; 22(2): 149-164.

[2] Pinter, P, Dietrich, S, Bertram, B, et al. Comparison and error estimation of 3D fibre orientation analysis of computed tomography image data for fibre reinforced composites. NDT \& E Int 2018; 95: 26-35.

[3] Meyer, N, Saburow, O, Hohberg, M, et al. Parameter identification of fiber orientation models based on direct fiber simulation with smoothed particle hydrodynamics. J Composites Sci 2020; 4(2): 77.

[4] Görthofer, J, Schneider, M, Ospald, F, et al. Computational homogenization of sheet molding compound composites based on high fidelity representative volume elements. Computat Mater Sci 2020; 174: 109456.

[5] Müller, V, and Böhlke, T. Prediction of effective elastic properties of fiber reinforced composites using fiber orientation tensors. Composites Sci Technol 2016; 130: 36-45.

[6] Kehrer, L, Wood, JT, and Böhlke, T. Mean-field homogenization of thermoelastic material properties of a long fiber-reinforced thermoset and experimental investigation. J Composite Mater 2020; 0021998320920695.

[7] Schemmann, M, Görthofer, J, Seelig, T, et al. Anisotropic meanfield modeling of debonding and matrix damage in SMC composites. Composites Sci Technol 2018; 161: 143-158.

[8] Cintra, JS Jr, and Tucker, CL III. Orthotropic closure approximations for flow-induced fiber orientation. J Rheol 1995; 39(6): $1095-1122$

[9] Chung, DH, and Kwon, TH. Invariant-based optimal fitting closure approximation for the numerical prediction of flow-induced fiber orientation. $J$ Rheol 2002; 46(1): 169-194.

[10] Cowin, SC. The relationship between the elasticity tensor and the fabric tensor. Mech Mater 1985; 4(2): $137-147$.

[11] Köbler, J, Schneider, M, Ospald, F, et al. Fiber orientation interpolation for the multiscale analysis of short fiber reinforced composite parts. Computat Mech 2018; 61(6): 729-750. 
[12] Köbler, J, Magino, N, Andrä, H, et al. A computational multi-scale model for the stiffness degradation of short-fiber reinforced plastics subjected to fatigue loading. Comput Meth Appl Mech Eng 2021; 373: 113522.

[13] Nomura, S, Kawai, H, Kimura, I, et al. General description of orientation factors in terms of expansion of orientation distribution function in a series of spherical harmonics. J Polym Sci A-2 Polym Phys 1970; 8(3): 383-400.

[14] Jack, DA, and Smith, DE. Elastic properties of short-fiber polymer composites, derivation and demonstration of analytical forms for expectation and variance from orientation tensors. J Composite Mater 2008; 42(3): 277-308.

[15] Breuer, K, Stommel, M, and Korte, W. Analysis and evaluation of fiber orientation reconstruction methods. J Composites Sci 2019; 3(3): 67.

[16] Advani, SG, and Tucker, CL III. Closure approximations for three-dimensional structure tensors. J Rheol 1990; 34(3): 367-386.

[17] Han, KH, and Im, YT. Modified hybrid closure approximation for prediction of flow-induced fiber orientation. $J$ Rheol 1999; 43(3): 569-589.

[18] Montgomery-Smith, S, He, W, Jack, DA, et al. Exact tensor closures for the three-dimensional Jeffery's equation. $J$ Fluid Mech 2011; 680: 321-335.

[19] Jack, D, and Smith, D. The effect of fibre orientation closure approximations on mechanical property predictions. Composites $A$ Appl Sci Manuf 2007; 38(3): 975-982.

[20] Buck, F, Brylka, B, Müller, V, et al. Two-scale structural mechanical modeling of long fiber reinforced thermoplastics. Composites Sci Technol 2015; 117: 159-167.

[21] Goldberg, N, Ospald, F, and Schneider, M. A fiber orientation-adapted integration scheme for computing the hyperelastic tucker average for short fiber reinforced composites. Computat Mech 2017; 60(4): 595-611.

[22] Böhlke, T. Application of the maximum entropy method in texture analysis. Computat Mater Sci 2005; 32(3-4): 276-283.

[23] Mandel, J. Généralisation de la théorie de plasticité de WT Koiter. Int J Solids Struct 1965; 1(3): 273-295.

[24] Harris, CR, Millman, KJ, van der Walt, SJ, et al. Array programming with NumPy. Nature 2020; 585(7825): 357-362.

[25] Meurer, A, Smith, CP, Paprocki, M, et al. Sympy: symbolic computing in Python. PeerJ Comput Sci 2017; 3: e103.

[26] Advani, SG, and Tucker, CL III. The use of tensors to describe and predict fiber orientation in short fiber composites. $J$ Rheol 1987; 31(8): 751-784.

[27] Görthofer, J, Meyer, N, Pallicity, TD, et al. Virtual process chain of sheet molding compound: Development, validation and perspectives. Composites B Eng 2019; 169: 133-147.

[28] Schemmann, M, Brylka, B, Gajek, S, et al. Parameter identification by inverse modelling of biaxial tensile tests for discontinous fiber reinforced polymers. PAMM 2015; 15(1): 355-356.

[29] Fernández, ML, and Böhlke, T. Representation of Hashin-Shtrikman bounds in terms of texture coefficients for arbitrarily anisotropic polycrystalline materials. J Elasticity 2019; 134(1): 1-38.

[30] Forte, S, and Vianello, M. Symmetry classes for elasticity tensors. J Elasticity 1996; 43(2): 81-108.

[31] Böhlke, T. Crystallographic Texture Evolution and Elastic Anisotropy: Simulation, Modeling, and Applications. Shaker, 2001.

[32] Francois, M, Geymonat, G, and Berthaud, Y. Determination of the symmetries of an experimentally determined stiffness tensor: Application to acoustic measurements. Int J Solids Struct 1998; 35(31-32): 4091-4106.

[33] Spencer, A. A note on the decomposition of tensors into traceless symmetric tensors. Int J Eng Sci 1970; 8(6): 475-481.

[34] Mentges, N, Dashtbozorg, B, and Mirkhalaf, S. A micromechanics-based artificial neural networks model for elastic properties of short fiber composites. Composites B Eng 2021; : 108736.

[35] Mehrabadi, MM, and Cowin, SC. Eigentensors of linear anisotropic elastic materials. QJ Mech Appl Math 1990; 43(1): 15-41.

[36] Dray, D, Gilormini, P, and Régnier, G. Comparison of several closure approximations for evaluating the thermoelastic properties of an injection molded short-fiber composite. Composites Sci Technol 2007; 67(7-8): 1601-1610.

[37] Backus, G. A geometrical picture of anisotropic elastic tensors. Rev Geophys 1970; 8(3): 633-671.

[38] Mochizuki, E. Spherical harmonic decomposition of an elastic tensor. Geophys J Int 1988; 93(3): 521-526.

[39] Baerheim, R. Harmonic decomposition of the anisotropic elasticity tensor. Q J Mech Appl Math 1993; 46(3): 391-418.

[40] Boehler, JP, Kirillov, A, and Onat, ET. On the polynomial invariants of the elasticity tensor. J Elasticity 1994; 34(2): 97-110.

[41] Olive, M, Kolev, B, and Auffray, N. A minimal integrity basis for the elasticity tensor. Arch Rat Mech Anal 2017; $226(1)$ : 1-31.

[42] Rychlewski, J. A qualitative approach to Hooke's tensors. Part I. Arch Mech 2000; 52(4-5): 737-759.

[43] Rychlewski, J. A qualitative approach to Hooke's tensors. Part II. Arch Mech 2001; 53(1): 45-63.

[44] Böhlke, T, and Brüggemann, C. Graphical representation of the generalized Hooke's law. Techn Mech 2001; 21(2): 145-158.

[45] Cowin, SC. Properties of the anisotropic elasticity tensor. Q J Mech Appl Math 1989; 42(2): 249-266.

[46] Musgrave, M. Crystal Acoustics. Holden-Day, 1970.

[47] Lobos Fernández, M. Homogenization and materials design of mechanical properties of textured materials based on zeroth-, first- and second-order bounds of linear behavior. In: Schriftenreihe Kontinuumsmechanik im Maschinenbauhanik, Vol. 12. KIT Scientific Publishing, 2018.

[48] Chung, DH, and Kwon, TH. Improved model of orthotropic closure approximation for flow induced fiber orientation. Polym Composites 2001; 22(5): 636-649.

[49] nilsmeyerkit, and JulianKarlBauer. nilsmeyerkit/fiberoripy: v1.0.12, 2021. Available at: https://doi.org/10.5281/zenodo.4679756.

[50] VerWeyst, BE, Tucker, C, Foss, PH, et al. Fiber orientation in 3-D injection molded features. Int Polym Process 1999; 14(4): 409-420. 
[51] Montgomery-Smith, S, Jack, D, and Smith, DE. The fast exact closure for Jeffery's equation with diffusion. J Non-Newtonian Fluid Mech 2011; 166(7-8): 343-353.

[52] Jack, DA, Schache, B, and Smith, DE. Neural network-based closure for modeling short-fiber suspensions. Polym Composites 2010; 31(7): 1125-1141.

\section{Appendix A. Material symmetries of second-order tensors}

Following [10], any symmetric tensor of second-order $\mathbf{A}$ is either isotropic, transversely isotropic, or orthotropic. This means, for symmetric second-order tensors, three material symmetry classes exist and representations of $\mathbf{A}$ in its material coordinate system $\left\{\mathbf{v}_{i}\right\}$ are given for the case of isotropy $\mathbf{A}^{\text {iso }}$, transversely isotropy $\mathbf{A}^{\text {transv }}$, and orthotropy $\mathbf{A}^{\text {ortho }}$ by

$$
\mathbf{A}^{\text {iso }}=a_{1}\left[\begin{array}{ccc}
1 & 0 & 0 \\
0 & 1 & 0 \\
0 & 0 & 1
\end{array}\right] \mathbf{v}_{i} \otimes \mathbf{v}_{j}, \quad \mathbf{A}^{\text {transv }}=\left[\begin{array}{ccc}
a_{1} & 0 & 0 \\
0 & a_{2} & 0 \\
0 & 0 & a_{2}
\end{array}\right] \mathbf{v}_{i} \otimes \mathbf{v}_{j}, \quad \mathbf{A}^{\text {ortho }}=\left[\begin{array}{ccc}
a_{1} & 0 & 0 \\
0 & a_{2} & 0 \\
0 & 0 & a_{3}
\end{array}\right] \mathbf{v}_{i} \otimes \mathbf{v}_{j} .
$$

The principle direction of the transversely isotropic case is randomly chosen to be the first axis. The number of independent coefficients is one, two, and three, respectively, and is indicated by the number of $a_{i}$.

\section{Appendix B. Parameter sets of specific second-order orientation tensors}

Table 2 contains arguments of parameterizations given in Equations (25), (28) and (30) leading to special orientation states.

Table 2. Arguments of parameterizations of $\mathbf{N}$ for special orientation states with $a \in \mathcal{R}_{*}^{+}$, i.e., $a>0$. Note: The orientation states labeled by planar isotropic $\mathbf{v}_{1}$ and planar isotropic $\mathbf{v}_{3}$, differ only by ordering convention of the eigenvalues and describe the same physical state.

\begin{tabular}{lllllllll}
\hline & $\begin{array}{l}\mathbf{N}\left(\lambda_{1}, \lambda_{2}\right) \\
\lambda_{1}\end{array}$ & $\lambda_{2}$ & $\begin{array}{l}\mathbf{N}\left(\alpha_{1}, \alpha_{3}\right) \\
\alpha_{1}\end{array}$ & $\alpha_{3}$ & $\begin{array}{l}\mathbf{N}(\hat{a}, \hat{c}) \\
\hat{a}\end{array}$ & $\hat{c}$ & \multicolumn{2}{c}{$\begin{array}{c}\mathbf{N}\left(\hat{w}_{1}, \hat{w}_{2}, \hat{w}_{2}\right) \\
\hat{w}_{1}\end{array}$} \\
\hline \hline isotropic & $1 / 3$ & $1 / 3$ & 0 & 0 & 0 & 0 & $a$ & $a$ \\
$\hat{w}_{2}$ & $\hat{w}_{3}$ \\
unidirectional $\mathbf{v}_{1}$ & 1 & 0 & $2 / 3$ & 0 & $2 / 3$ & $-1 / 3$ & $a$ & 0 \\
planar isotropic $\mathbf{v}_{1}$ & 0 & $1 / 2$ & $-1 / 3$ & 0 & $-1 / 3$ & $1 / 6$ & 0 & $a$ \\
planar isotropic $\mathbf{v}_{3}$ & $1 / 2$ & $1 / 2$ & 0 & $-1 / 3$ & $1 / 6$ & $-1 / 3$ & $a$ & $a$ \\
\hline
\end{tabular}

\section{Appendix C. Coefficient-wise extrema of moment tensors}

Given a triangulation which divides the unit sphere into $m$ surfaces $\mathcal{F}_{\gamma}$ with centered normal vectors $\mathbf{n}_{\gamma}$ for $\gamma \in[1, \ldots, m]$. The integration over $\mathcal{S}^{2}$ in Equation (10) can be interpreted as a weighted summation with discrete weights $w_{\gamma}$ derived by the ODF by

$$
w_{\gamma}=\int_{\mathcal{F}_{\gamma}} \psi(\mathbf{n}) \underline{n} .
$$

The properties of $\psi(\mathbf{n})$ imply non-negative and normalized weights, i.e., $0 \leq w_{\gamma}$ and $\sum_{\gamma}^{m} w_{\gamma}=1$ holds. Definition of $\mathbb{N}$ in Equation (10) is the limiting case

$$
\mathbb{N}=\lim _{m \rightarrow \infty} \sum_{\gamma}^{m} w_{\gamma} \mathbf{n}_{\gamma}^{\otimes 4}
$$

Defining coefficients of the $\gamma$ th moment tensor by $\mathbf{n}_{\gamma}^{\otimes 4}=\left(n_{\gamma}^{\otimes 4}\right)_{\xi \zeta} \mathbf{B}_{\xi} \otimes \mathbf{B}_{\zeta}$, Equation (95) reads

$$
N_{\xi \zeta}^{(4)}=\lim _{m \rightarrow \infty} \sum_{\gamma}^{m} w_{\gamma}\left(n_{\gamma}^{\otimes 4}\right)_{\xi \zeta} \text {. }
$$


The set of weights $\left\{w_{\gamma}: 0 \leq w_{\gamma}, \sum_{\gamma}^{m} w_{\gamma}=1\right\}$ describes an $m$-dimensional region $\mathcal{W}$ and $\mathbb{N}$ is linear in these weights. Due to the linearity, the extreme values of $\mathbb{N}$ arise at the boundary of $\mathcal{W}$, especially at points where only one weight equals one and the remaining weights are zero. Such points are corners of the boundary of $\mathcal{W}$. As

$$
\sum_{\gamma}^{m} w_{\gamma}\left[\left(n_{\gamma}^{\otimes 4}\right)_{\xi \zeta}-\left(n_{\min }^{\otimes 4}\right)_{\xi \zeta}\right] \geq 0
$$

is valid it follows from

$$
\begin{aligned}
\sum_{\gamma}^{m} w_{\gamma}\left(n_{\gamma}^{\otimes 4}\right)_{\xi \zeta}-\left(n_{\min }^{\otimes 4}\right)_{\xi \zeta} & \geq 0 \\
\sum_{\gamma}^{m} w_{\gamma}\left(n_{\gamma}^{\otimes 4}\right)_{\xi \zeta} & \geq\left(n_{\min }^{\otimes 4}\right)_{\xi \zeta}
\end{aligned}
$$

that the minimum of a coefficient of a moment tensor $\left(n_{\min }^{\otimes 4}\right)_{\xi \zeta}$ is a lower bound for the corresponding coefficient of the fourth-order orientation tensor $N_{\xi \zeta}^{(4)}$. Note, that for a fixed coordinate system, there exists a singular ODF $\psi^{\min }(\mathbf{n})$ of shape

$$
\psi^{\min }(\mathbf{n})=\frac{1}{2}\left(\delta\left(\mathbf{n}-\mathbf{n}^{*}\right)+\delta\left(\mathbf{n}+\mathbf{n}^{*}\right)\right)
$$

leading to $N_{\xi \zeta}^{(4)}=\left(n_{\min }^{\otimes 4}\right)_{\xi \zeta}$. Similar reasoning leads to coefficient-wise upper limits of $N_{\xi \zeta}^{(4)}$ defined by maximum coefficients of a moment tensor $\left(n_{\max }^{\otimes 4}\right)_{\xi \zeta}$ corresponding to a singular ODF. As a consequence, extreme values of tensor coefficients of $\mathbb{N}$ can be derived from the moment tensor $\mathbf{n}^{\otimes 4}$. However, this coefficient-wise consideration neglects the interaction of the coefficients of $\mathbb{N}$ and the procedure defining the orientation coordinate system $\left\{\mathbf{v}_{i}\right\}$. Tensor coefficients of a representation in a coordinate system, which depends, e.g., on the eigenvectors of $\mathbf{N}$, may not reach the extreme values reachable in a fixed coordinate system. Another consequence is that the norm of $\mathbb{N}$ is bound by the value one, which is reached for a singular ODF. Parameterization of $\mathbf{n}^{\otimes 4}$ in two angles with

$$
\mathbf{n}(\theta, \varphi)=\sin (\theta) \cos (\varphi) \mathbf{e}_{1}+\sin (\theta) \sin (\varphi) \mathbf{e}_{2}+\cos (\theta) \mathbf{e}_{3}
$$

leads to a representation of the moment tensor by $\mathbf{n}^{\otimes 4}=n_{\xi \zeta}^{\otimes 4} \mathbf{B}_{\xi} \otimes \mathbf{B}_{\zeta}$ with

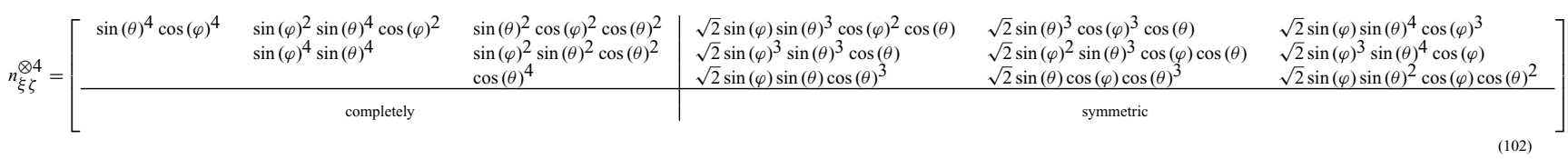

having extreme coefficient-wise values

$$
\underbrace{\left[\begin{array}{lll|lll}
0 & 0 & 0 & -b & -a & -a \\
& 0 & 0 & -a & -b & -a \\
& 0 & -a & -a & -b \\
\hline \multirow{2}{*}{\text { completely }} & \text { symmetric }
\end{array}\right]}_{=} \leq n_{\xi \zeta}^{\otimes 4} \leq n_{\xi \zeta}^{\otimes 4}
$$


with $a=\sqrt{2} \frac{3 \sqrt{3}}{16}$ and $b=\frac{\sqrt{2}}{8}$. Exemplary application of the coefficient-wise limits on transversely isotropic fourth-order orientation tensors defined in Equation (64), leads to the set

$$
\begin{aligned}
\mathcal{N}_{\text {linear }}^{\text {transv }}= & \left\{\mathbb{N}^{\text {transv }} \mid \min _{\phi, \theta} n_{\xi \zeta}^{\otimes 4} \leq N_{\xi \zeta}^{(4)} \leq \max _{\phi, \theta} n_{\xi \zeta}^{\otimes 4}\right\} \\
= & \left\{\mathbb{N}^{\text {transv }}(\alpha, \rho) \mid-\frac{1}{3} \leq \alpha \leq \frac{2}{3},\right. \\
& \left.\quad \max \left(\frac{1}{280}(-30 \alpha-7), \frac{1}{105}(15 \alpha-7)\right) \leq \rho \leq \frac{1}{840}(15 \alpha+14)\right\}
\end{aligned}
$$

which is visualized in Figure 3.

\section{Appendix D. Parameterization of admissible $\mathbb{N}^{\text {ortho }}$ with isotropic $\mathbf{N}$}

Explicit parameterization of those fourth-order orientation tensors which contract to the isotropic second-order orientation tensor is given by the set

$$
\begin{aligned}
\left\{\mathbb{N}^{\text {ortho }}\left(\mathbf{N}^{\text {iso }}, d_{1}, d_{2}, d_{3}\right) \mid-\frac{1}{15}\right. & \leq d_{1} \leq \frac{1}{10} \\
-\frac{1}{15} & \leq d_{2} \leq \frac{7}{45}+\frac{5}{9\left(45 d_{1}-7\right)} \\
-\frac{1}{15} & \left.\leq d_{3} \leq \frac{4-60 d_{1}-60 d_{2}+675 d_{1} d_{2}}{60-675 d_{1}-675 d_{2}}\right\} \subset \mathcal{N}^{\text {ortho }} .
\end{aligned}
$$

